\title{
An elite broadly neutralizing antibody protects SARS-CoV-2 Omicron
}

\section{Authors:}

5 Biao Zhou ${ }^{1,2,8}$, Runhong Zhou ${ }^{1,2,8}$, Jasper Fuk-Woo Chan ${ }^{2,3,4,5,6,7,8}$, Mengxiao Luo ${ }^{1,2,8}$, Qiaoli

6 Peng $^{1,2,8}$, Shuofeng Yuan ${ }^{2,3,4,5,6,8}$, Bobo Wing-Yee Mok ${ }^{2,3,4}$, Bohao Chen ${ }^{1,2}$, Pui Wang ${ }^{2,3,4}$,

7 Vincent Kwok-Man Poon ${ }^{2}$, Hin $\mathrm{Chu}^{2,3,4}$, Chris Chung-Sing Chan ${ }^{2}$, Jessica Oi-Ling Tsang ${ }^{2}$,

8 Chris Chun-Yiu Chan ${ }^{2}$, Ka-Kit Au ${ }^{1,2}$, Hiu-On Man ${ }^{1,2}$, Lu Lu², Kelvin Kai-Wang To ${ }^{2,3,4,5,6}$,

9 Honglin Chen ${ }^{2,3,4,6}$, Kwok-Yung Yuen ${ }^{2,3,4,5,6,7}$ and Zhiwei Chen ${ }^{1,2,3,4,6 \text { 区 }}$

\section{Affiliations:}

$12{ }^{1}$ AIDS Institute, Li Ka Shing Faculty of Medicine, The University of Hong Kong, Pokfulam, 13 Hong Kong Special Administrative Region, People's Republic of China.

$14{ }^{2}$ Department of Microbiology and Carol Yu Centre for Infection, Li Ka Shing Faculty of 15 Medicine, The University of Hong Kong, Pokfulam, Hong Kong SAR, People's Republic of 16 China.

$17{ }^{3}$ State Key Laboratory of Emerging Infectious Diseases, The University of Hong Kong, 18 Pokfulam, Hong Kong SAR, People's Republic of China.

$19{ }^{4}$ Centre for Virology, Vaccinology and Therapeutics, Health@InnoHK, The University of 20 Hong Kong, Hong Kong Special Administrative Region, Hong Kong, People's Republic of 21 China.

$22{ }^{5}$ Department of Clinical Microbiology and Infection Control, The University of Hong Kong-

23 Shenzhen Hospital, Shenzhen, Guangdong, People's Republic of China.

$24{ }^{6}$ Department of Microbiology, Queen Mary Hospital, Pokfulam, Hong Kong Special

25 Administrative Region, China.

$26{ }^{7}$ Academician Workstation of Hainan Province and Hainan Medical University-The 27 University of Hong Kong Joint Laboratory of Tropical Infectious Diseases, The University of

28 Hong Kong, Pokfulam, Hong Kong Special Administrative Region, China.

$29{ }^{8}$ These authors made equal contributions as co-first authors.

30 घCorrespondence to: zchenai@hku.hk 


\section{ABSTRACT}

32 The strikingly high transmissibility and antibody evasion of SARS-CoV-2 Omicron variant

33 have posted great challenges on the efficacy of current vaccines and antibody immunotherapy.

34 Here, we screened 34 BNT162b2-vaccinees and cloned a public broadly neutralizing 35 antibody (bNAb) ZCB11 from an elite vaccinee. ZCB11 neutralized all authentic SARS-

$36 \mathrm{CoV}-2$ variants of concern (VOCs), including Omicron and Omicron ${ }_{\mathrm{R} 346 \mathrm{~K}}$ with potent $\mathrm{IC}_{50}$

37 concentrations of 36.8 and $11.7 \mathrm{ng} / \mathrm{mL}$, respectively. Functional analysis demonstrated that

38 ZCB11 targeted viral receptor-binding domain (RBD) and competed strongly with ZB8, a

39 known RBD-specific class II NAb. Pseudovirus-based mapping of 57 naturally occurred

40 single mutations or deletions revealed that only S371L resulted in 11-fold neutralization

41 resistance, but this phenotype was not observed in the Omicron variant. Furthermore,

42 prophylactic ZCB11 administration protected lung infection against both the circulating

43 pandemic Delta and Omicron variants in golden Syrian hamsters. These results demonstrated

44 that vaccine-induced ZCB11 is a promising bNAb for immunotherapy against pandemic

45 SARS-CoV-2 VOCs.

\section{Keywords}

48 SARS-CoV-2; COVID-19; Variants of concern; Broadly neutralizing antibody; Omicron;

49 Delta; Golden Syrian hamster 


\section{INTRODUCTION}

54 After two years of the COVID-19 pandemic, the highly transmissible SARS-CoV-2 and its 55 variant of concerns (VOCs) have resulted in more than 279 million infections with 5.4 56 million deaths globally by December 26, 2021 (https://coronavirus.jhu.edu/map.html).

57 During this period, various types of COVID-19 vaccines have been quickly developed to 58 control the pandemic with over 8.9 billion doses administered in many countries. Although 59 the extensive implementation of vaccination has significantly reduced the rates of 60 hospitalization, severity and death ${ }^{1-5}$, current vaccines do not confer complete or durable 61 prevention of upper airway transmission of SARS-CoV-2. The numbers of vaccine62 breakthrough infections and re-infections, therefore, have been continuously increasing ${ }^{6-8}$. 63 The pandemic situation has been complicated by repeated emergence of new VOCs, 64 including Alpha (B.1.1.7), Beta (B.1.351), Gamma (P.1), Delta (B.1.617.2) and Omicron 65 (B.1.1.529) ${ }^{9,10}$, and waning of vaccine-induced immune responses, together with relaxed 66 preventive masking and social distancing ${ }^{11-13}$.

68 After the World Health Organization (WHO) designated the Omicron as a VOC on 69 November 26, 2021, this variant has been quickly found in over 110 countries and is 70 replacing the Delta VOC within a month, becoming the dominant VOC in many places in the 71 South Africa, European countries, and the United States ${ }^{14,15}$. According to the GASAID 72 database, for example, the relative variant genome frequency of the current circulating Delta 73 VOC has declined from $89 \%$ to $19.6 \%$ while the Omicron VOC has increased from $0 \%$ to $7467.4 \%$ in African countries during the period from October 4, 2021, to December 26, 2021. 75 The rapid global spread of the Omicron VOC has been associated with vaccine-breakthrough 76 infections and re-infections 16,17. Moreover, like previous findings that the Beta VOC 77 compromised vaccine-induced neutralizing antibody (NAb) ${ }^{12,18,19}$, the Omicron VOC has 78 resulted in even worse NAb evasion due to more than 30 alarming mutations in SARS-CoV-2 79 spike glycoprotein ${ }^{20-23}$. Considering that current NAb combination for clinical 80 immunotherapy showed significantly reduced activities ${ }^{21,24}$, we sought to search for vaccine81 induced broadly neutralizing antibody (bNAbs) among elite vaccinees.

\section{RESULTS}


86 To isolate potent bNAbs against currently circulating SASR-CoV-2 VOCs, we searched for

87 elite vaccinees, who had developed potent bNAbs among a Hong Kong cohort of 34 88 vaccinees, around average 30.7 days (range, 7-47 days) after their second dose of the 89 BNT162b2 vaccination (BioNTech-Pfizer) (Supplementary Table 1) ${ }^{13}$. 100\% subjects 90 developed NAbs against the pseudotyped SARS-CoV-2 wildtype (WT, D614G) (Fig. 1a, top 91 left). To seek for vaccinees with bNAb, we then tested the full panel of pseudotyped SARS92 CoV-2 VOCs including Alpha (B.1.1.7), Beta (B.1.351), Gamma (P.1), Delta (B.1.617.2) and 93 Omicron (B.1.1.529). Only two study subjects (2/34), BNT162b2-26 and BNT162b2-55, 94 were considered as elite vaccinees who harbored bNAbs with $\mathrm{IC}_{90}$ or $\mathrm{IC}_{50}$ values higher than 95 the mean titers of all VOCs tested in the cohort. BNT162b2-26 displayed significantly high 96 bNAbs titers against the Beta and Delta variants (Fig. 1a, mid left and bottom left, 97 Supplementary Table 2), the known most resistant VOC and the dominant VOC, 98 respectively, before the Omicron variant ${ }^{25,26}$. After measuring binding antibodies to spike 99 protein (Fig. 1b), we calculated the neutralizing potency index as previously described ${ }^{27}$. We 100 found that Omicron resulted in the highest reduction of the mean neutralizing potency index 101 as compared with other VOCs (Fig. 1c). BNT162b2-26, however, displayed neutralizing 102 potency index scores consistently higher than the mean ones against all VOCs tested. We, 103 therefore, chose this elite vaccinee for subsequent search of bNAb.

\section{Isolation of NAbs against SARS-CoV-2 from the elite vaccinee}

106 With vaccinee informed consent, we obtained another blood sample donated by BNT162b210726 at day 130 after his second vaccination. Fresh PBMCs from BNT162b2-26 were stained 108 for antigen-specific memory B cells (CD19, CD27, IgG) using the 6xHis-tagged SARS-CoV1092 WT spike as the bait as previously described ${ }^{28}$. Spike-specific memory B cells were found 110 in BNT162b2-26 but not in the healthy donor (HD) control (Supplementary Fig. 1) and were 111 sorted into each well with a single B cell for antibody gene amplification. After antibody 112 gene sequencing, we recovered 14-paired heavy chain and light chain for antibody IgG1 113 engineering. Seven of these 14 paired antibodies including ZCB3, ZCB8, ZCB9, ZCB11, 114 ZCC10, ZCD3, ZCD4 in antibody expression supernatants showed positive responses to WT 115 spike by ELISA 48 hours post transient transfection (Supplementary Fig. 2a). Five of these 116 seven spike-reactive antibodies including ZCB3, ZCD4, ZCB11, ZCC10 and ZCD3 targeted 117 spike S1 subunit (Supplementary Fig. 2b), whereas ZCB8 and ZCB9 were S2-specific 118 (Supplementary Fig. 2c). Moreover, among these five S1-reactive antibodies, only ZCD4 was 119 not specific to RBD (Supplementary Fig. 2d) and none of them interacted with NTD 
120 (Supplementary Fig. 2e, Supplementary Table 3). Eventually, only four RBD-specific ZCB3,

121 ZCB11, ZCC10 and ZCD3 showed neutralizing activities against WT by the pseudovirus 122 neutralization assay (Supplementary Fig. 2f). These results demonstrated that RBD-specific 123 NAbs were primarily obtained from memory B cells of BNT162b2-26 at 130 days after his 124 second vaccination.

126 Notably, besides the previously published control ZB8 ${ }^{28}$, ZCB11 had the strongest binding 127 capability to both RBD and Spike with the same $\mathrm{EC}_{50}$ values of $20 \mathrm{ng} / \mathrm{ml}$ by ELISA (Fig. 2a, 128 Supplementary Table 5). Moreover, the binding dynamics of ZCB11 to SARS-CoV-2 RBD 129 was determined using the surface plasmon resonance (SPR). We found that ZCB11 exhibited 130 the fast-on/slow-off kinetics with an equilibrium dissociation constant (KD) value of $1315.75 \times 10^{-11} \mathrm{M}$, suggesting an RBD-specific high-binding affinity (Supplementary Fig. 2g and 132 Supplementary Table 6). In subsequent quantitative neutralization analysis against WT, we 133 found that two of these four NAbs, ZCB3 and ZCB11, showed high neutralization potency 134 with $\mathrm{IC}_{50}$ values below $100 \mathrm{ng} / \mathrm{mL}$ (Fig. 2b top left, Supplementary Table 7). Sequence 135 analysis revealed that ZCB3, ZCC10 and ZCD3 utilized IGHV3-53/3-66 heavy chain, 136 whereas their paired light chains had distinct IGKV1-9, IGKV3-20 and IGKV1-27, 137 respectively (Supplementary Table 3). In contrast, ZCB11 utilized different IGHV1-58 heavy 138 chain and IGKV3-20 light chain. Our four new NAbs were all considered as public 139 antibodies characterized by a IGHV3-53/3-66 heavy chain with 10-12 residues in the CDR3 140 region or a IGHV1-58 heavy chain with 15-17 residues in CDR3 region as previous reported 141 by others ${ }^{12,29,30}$. These results demonstrated BNT162b2-26 developed mainly public NAbs 142 after two doses of vaccination.

\section{Antibody neutralization of SARS-CoV-2 VOCs}

145 To understand the breadth of these four newly cloned public RBD-specific NAbs, we 146 performed SARS-CoV-2 neutralization assays using both pseudoviruses and authentic VOC 147 isolates, including Alpha, Beta, Gamma, Delta and Omicron variants (Fig. 2b). ZB8, a known 148 RBD-specific class II NAb, was included as a positive control. Testing pseudoviruses in 149 293T-ACE2 cells, we found that ZCB11 was the best bNAb that neutralized all VOCs 150 potently, including the most alarming Omicron variant ${ }^{21}$, with $\mathrm{IC}_{50}$ values of around 30 $151 \mathrm{ng} / \mathrm{mL}$ for Gamma and Delta variants and $6 \mathrm{ng} / \mathrm{mL}$ for Alpha, Beta and Omicron variants 152 (Fig. 2b, Supplementary Table 7). ZCB3 was the second best bNAb and neutralized Alpha, 153 Beta, Gamma and Delta variants potently, but not the Omicron variant. ZCC10 and ZCD3 
154 neutralized Alpha, Gamma and Delta variants at relative low potency, but lost neutralization 155 totally against Beta and Omicron variants. Importantly, testing authentic VOC viruses in 156 Vero-E6-TMPRSS2 cells, we consistently found that ZCB11 was the most potent bNAb, 157 followed by ZCB3 (Fig. 2c). The IC $_{50}$ values of ZCB11 for neutralizing Alpha, Beta, Gamma, 158 Delta, Omicron and Omicron $_{\mathrm{R} 346 \mathrm{~K}}$ variants were 85.1, 39.9, 56.9, 11.2, 36.8 and $11.7 \mathrm{ng} / \mathrm{mL}$, 159 respectively, which were comparable to the $\mathrm{IC}_{50}$ value of $51 \mathrm{ng} / \mathrm{ml}$ for neutralizing the WT 160 (Supplementary Table 7). ZCB3 was about 10-fold less potent than ZCB11 for neutralizing 161 Beta and Omicron variants. Notably, the potency of ZCB11 in the pseudovirus assay was 162 higher than that in the authentic virus assay, which was probably related to different target 163 cells used. ZB8 showed unmeasurable and weak neutralization against Delta pseudovirus and 164 Delta authentic virus, respectively. Conversely, ZCC10 and ZCD3 showed weak and 165 unmeasurable neutralization against Gamma pseudovirus and Gamma authentic virus, 166 respectively. These results demonstrated that ZCB11 functioned as an elite bNAb potently 167 neutralized all circulating SARS-CoV-2 VOCs in vitro. Notably, although BNT162b2-26 168 developed mainly public NAbs, ZCB11 was unlikely dominantly elicited due to the reduced 169 titer against Omicron as compared with WT (Fig. 1).

\section{Naturally occurred mutations or deletions conferring antibody resistance}

172 Since Omicron variant escaped from NTD-specific NAbs and majority of known RBD-

173 specific NAbs in the class I, class II, class III and class IV groups ${ }^{20,21,31}$, we sought to 174 determine possible mutations or deletions responsible for antibody resistance for ZCB3 and 175 ZCB11 as compared with the control ZB8. We first constructed and tested a large panel of 176 pseudoviruses carrying individual mutations or deletions found in Omicron variant as 177 compared with those previously found in Alpha, Beta, Gamma and Delta variants (Fig. 3a). 178 For ZB8, we consistently found that the E484 is essential for its neutralization activity. 179 E484K in Beta, E484Q in Delta and E484A in Omicron were responsible for the significant 180 ZB8 resistance, followed Q493R for about 10-fold resistance. For ZCB3, none of single 181 mutations or deletions tested conferred resistance for equal to or more than 10-fold. Only and 182 Q493R in Omicron reduced neutralization potency of around 3.5-fold. For ZCB11, only 183 S371L in Omicron showed 11.2-fold resistance (Fig. 3a). Moreover, Q493R, Y505H, T547K 184 and Q954H in Omicron exhibited over 6-fold resistance. Unexpectedly, when all these and 185 other mutations combined in Omicron, they did not confer significant resistance at all. 186 Subsequently, we performed antibody competition by Surface SPR. Although they engaged 187 different clonotype and antibody resistant profiles, ZCB11 exhibited as a strong competitor 
188 for WT RBD binding against either ZCB3 or ZB8, respectively (Fig. 3b and Supplementary

189 Fig. 3), suggesting overlapped antibody binding epitopes in RBD between them. To further 190 predict the binding mode of ZCB11, we searched structural database for RBD-specific NAbs 191 with similar B cell clonotype. Interestingly, the patient-derived S2E12 Nab, which used the 192 same IGHV1-58 heavy chain and IGKV3-20 light chain ${ }^{32}$, shared the high amino acid 193 identity of $82.2 \%$ in heavy chain variable regions with ZCB11. A model of ZCB11 variable 194 regions was generated based on the protein sequence by the SWISS-MODEL using the 195 crystal structure of S2E12 Fab fragment (Research Collaboratory for Structural 196 Bioinformatics [RCSB] PDB code 7K3Q) as the template. The superimposed ZCB11 and 197 S2E12 variable regions (Fig. 3c) showed that the secondary elements and most of loops are relatively conserved, except for the HCDR1 and KCDR3 which contained a single amino acid insertion and deletion, respectively. It is possible that ZCB11 also recognized the convex receptor binding motif (RBM) like S2E12. S477N, Q493R and Y505H mutations that conferred partial ZCB11 resistance in the pseudovirus assay were close to the binding interface between S2E12 and RBM (Fig. 3d).

203

\section{In vivo efficacy of ZCB11 against SARS-CoV-2 Delta and Omicron variants}

205 To determine the in vivo potency of ZCB11 against the dominant circulating VOCs, we conducted viral challenge experiments using the golden Syrian hamster COVID-19 model as compared with ZB8 ${ }^{33}$. Since ZB8 conferred nearly complete lung protection against SARSparallel with ZCB11 using the same dose according to our standard experimental procedure (Fig. 4a). One day prior vial challenge, three groups of hamsters $(n=8)$ received the intraperitoneal injection of ZCB11, ZB8 and PBS, respectively. Twenty-four hours later, half of the animals $(n=4)$ in each group were separated into subgroups and were challenged intranasally with $10^{5}$ PFU of SARS-CoV-2 Delta variant and Omicron variant, respectively. Animal body weight changes were measured daily until day 4 when all animals were sacrificed for endpoint analysis. For three subgroups challenged with the SASR-CoV-2 Delta variant, we found that the infection caused around $10 \%$ body weight loss overtime in the PBS and ZB8 pre-treatment groups. In contrast, transient and less than $4 \%$ body weight decrease

218 was observed for the ZCB11-treated hamsters (Fig. 4b). Moreover, relatively lower sub219 genomic viral loads (Fig. 4c) and unmeasurable numbers of live infectious viruses (six orders 220 of magnitude drop) (Fig. 4d) were achieved by ZCB11 than by ZB8. For hamsters challenged with the SASR-CoV-2 Omicron variant, no significant body weight loss was found in all 
222 three subgroups, indicating relatively weaker pathogenicity caused by Omicron than by Delta

223 (Fig. 4e). However, significantly lower sub-genomic viral loads (Fig. 4f) and unmeasurable 224 numbers of live infectious viruses (Fig. $4 \mathrm{~g}$ ) were achieved only by ZCB11. These results 225 demonstrated that ZCB11 conferred significant protection against both Delta and Omicron 226 variants, whereas ZB8 exhibited only partial protection against Delta but not Omicron. These 227 findings were consistent with in vitro neutralizing activities of ZCB11 and ZB8 against live 228 Delta and Omicron variants, respectively (Fig. 2c). Since the number of infectious viruses in the PBS group of Delta-challenged hamsters was over one order of magnitude higher than 230 that in the PBS group of Omicron-challenged animals (Fig. 4d and 4g), higher amount of 231 ZCB11 might be needed for improved suppression of sub-genomic viral loads against the Delta variant.

\section{DISCUSSION}

It remains unclear what type of human monoclonal NAbs can potently neutralize all current SARS-CoV-2 VOCs including the Omicron Variant. In this study, we showed that the standard two-dose BNT162b2 vaccination was able to induce spike-specific memory B cells, from which we successfully cloned the elite bNAb ZCB11 around 130 days post the second vaccination. We demonstrated that ZCB11 not only neutralized all authentic SARS-CoV-2 VOCs including Omicron and Omicron $_{\mathrm{R} 346 \mathrm{~K}}$ at comparable high potency in vitro but also protected golden Syrian hamsters against the major circulating Omicron and Delta variants.

242 Till now, few existing NAbs under clinical development have displayed similar neutralization

243 breadth and in vivo potency ${ }^{20,21}$. Since sequence analysis revealed that ZCB11 was a family 244 member of public antibodies with the IGHV1-58 heavy chain and IGKV3-20 light chain, our 245 findings have significant implication to vaccine design for inducing high amounts of ZCB11246 like bNAb for broad protection and for clinical development of ZCB11-based 247 immunotherapy against the pandemic SARS-CoV-2 VOCs.

249 ZCB11 overcomes naturally occurred spike mutations and deletions across current SARS250 CoV-2 VOCs. Alpha variant with D614G and N501Y mutations enhanced RBD binding to 251 human ACE2 receptor, transforming it into the most prevalent variant at the early stage of $2522021^{35}$. N501Y alone was found conferring partial resistance to RBD-specific class I 910-30 253 and NTD-specific 4-18 NAbs ${ }^{21}$. Subsequently, Beta, Gamma and Delta variants displayed 254 the most troublesome mutations including K417N, E484K/Q/A and N501Y, conferring high 255 resistance to RBD-specific class I and class II NAbs ${ }^{21,36-38}$. E484K/Q/A led to almost 
256 complete loss of neutralization by potent RBD-specific class II LY-CoV555 and 2-15 ${ }^{21}$.

257 Attributed probably by antibody evasion, Delta variant, carrying

258 L452R/T478K/D614G/P681R mutations, were found in more than 170 countries and 259 accounted for $99 \%$ of newly confirmed cases before the Omicron variant ${ }^{39-41}$. After the 260 emergence of the Omicron variant with more than 30 mutations in viral spike protein ${ }^{42}$, the 261 ongoing wave of COVID-19 pandemic has already been dominated by it over the Delta 262 variant in many countries probably due to further antibody evasion to almost all current 263 vaccines and NAbs including those approved for clinical use or emergency use ${ }^{11,21,31,43}$. 264 N440K and G446S in Omicron conferred resistance to class III antibodies such as 265 REGN10987 and 2-7 ${ }^{21}$. G142D and del143-145 led to resistance to NTD-specific 4-18 and 266 5-7, whereas S371L conferred much broader resistance to RBD-specific class I, class III and 267 class IV NAbs including potent Brii-196, REGN10987 and Brii-198 in clinical development $268{ }^{21}$. In this study, we consistently found that E484K/Q/A in Beta, Delta and Omicron variants 269 conferred strong resistance to our RBD-specific class II ZB8 NAb. These resistant mutations, 270 however, did not affect the potency of ZCB11 significantly. Although S371L in Omicron 271 displayed partial resistance $(\sim 11$-fold) to ZCB11, similar amount of resistance was not

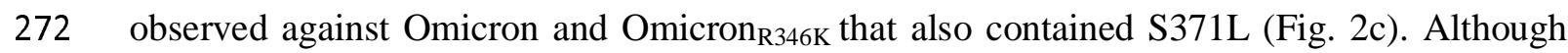
273 ZCB11 shared $86.5 \%$ amino acid identity in variable regions with the previously reported 274 ultrapotent S2E12 bNAb ${ }^{32,44}$, it is critical to solve the real structure of RBD-ZCB11 Fab 275 complex in future studies to understand if ZCB11 and S2E12 use an identical mode of action, 276 which will be useful for novel vaccine design to elicit ZCB11-like bNAb responses.

278 Most public antibodies were RBD-specific class I NAbs ${ }^{21,29,30,45,46}$. Accordingly, public 279 antibody is encoded by B cell clonotypes isolated from different individuals that share similar 280 genetic features ${ }^{47}$. In a previous study, 7 of 13 NAbs were found using IGHV3-53/3-66 281 heavy chain and paired predominantly with IGKV1-9*01 light chain ${ }^{21}$. These NAbs 282 displayed abolished neutralizing activity after $\mathrm{K} 417 \mathrm{~N}$ in Beta variant was introduced into the 283 pseudovirus neutralization assay. Interestingly, our newly cloned NAbs ZCB3, ZCC10 and 284 ZCD3 utilized the same IGHV3-53/3-66 heavy chain but paired with IGKV1-9, IGKV3-20 285 and IGKV1-27 light chains, respectively (Supplementary Table 4). ZCB3, our second best 286 bNAb, used the identical pair of IGHV3-53/3-66 and IGKV1-9 but did not display 287 neutralization reduction against the K417N pseudovirus. ZCB3, however, showed reduced 288 neutralization potency for over 10-fold against Beta and Omicron variants as compared with 289 ZCB11. More interestingly, our elite bNAb ZCB11 used IGHV1-58 heavy chain and IGKV3- 
29020 light chain, which also belongs to public antibodies reported by other groups ${ }^{29,32,47,48}$. In

291 these studies, patient-derived S2E12 and vaccine-induced 2C08 NAbs that shared 95\% amino

292 acid identity also used the same IGHV1-58 heavy chain and IGKV3-20 light chain. 2C08 was

293 able to prevent challenges against Beta and Delta variants in the hamster model. Like 82.2\%

294 amino acid identity between ZCB11 and S2E12, ZCB11 and 2C08 shared $83.8 \%$ amino acid

295 identity in their heavy chain variable regions. Their potency difference for neutralizing

296 Omicron remained to be determined. Nevertheless, vaccine design in eliciting high amounts

297 of ZCB11-like bNAb should be considered as a research priority, especially after its

298 clonotype has been found in different ethnic human populations but have not been abundantly

299 induced by current vaccines. Since ZCB11 protected hamsters against both the Delta and

300 Omicron variants, the most dominant circulating SARS-CoV-2 VOCs in the world, our

301 findings warrant the clinical development of ZCB11 and ZCB11-like bNAbs for patient

302 immunotherapy and transmission prevention.

303

\section{Limitations of the study}

305 ZCB11 probably represents the broadest breadth among bNAbs reported thus far with

306 comparable potency against all current SARS-CoV-2 VOCs including Omicron and

307 Omicron $_{\mathrm{R} 346 \mathrm{~K}}$. We are still in the process in determining its mode of action by solving

308 structures of the RBD-ZCB11 Fab complex. Such information will be useful to guide vaccine

309 design as mentioned because the frequency of elite vaccine remains low (2/34 in this study).

310 To understand the frequency of ZCB11-like bNAb among BNT162b2-vaccinees, we need to

311 investigate other elite responders who show equally potent bNAb responses. More ZCB11-

312 like bNAbs should be also discovered to improve current antibody-based cocktail

313 immunotherapy. For animal challenge experiments, we have done a single dose efficacy

314 experiment. Different doses and routes of administration or antibody combination will be

315 tested in future experiments to provide useful information to support clinical development of

316 ZCB11 and ZCB11-like bNAb. 


\section{METHODS}

\section{Human subjects}

319 A cohort of 34 vaccinees who received two doses of BNT162b2 before June 2021 were 320 recruited for this study. The exclusion criteria include individuals with (1) documented 321 SARS-CoV-2 infection, (2) high-risk infection history within 14 days before vaccination, (3) 322 COVID-19 symptoms such as sore throat, fever, cough and shortness of breath. Clinical and 323 laboratory findings were entered into a predesigned database. Written informed consent was 324 obtained from all study subjects. This study was approved by the Institutional Review Board 325 of The University of Hong Kong/Hospital Authority Hong Kong West Cluster (Ref No. UW 326 21-120 452).

Viruses

329 Authentic SARS-CoV-2 D614G (MT835143), Alpha (MW856794), Beta (GISAID: 330 EPI_ISL_2423556), Omicron (hCoV-19/Hong Kong/HKU-344/2021; GISAID accession 331 number EPI_ISL_7357684) and Delta (hCoV-19/Hong Kong/HKU-210804-001/2021; 332 GISAID: EPI_ISL_3221329) variants were isolated from respiratory tract specimens of 333 laboratory-confirmed COVID-19 patients in Hong Kong ${ }^{24}$. All experiments involving live 334 SARS-CoV-2 followed the approved standard operating procedures of the Biosafety Level 3 335 facility at The University of Hong Kong ${ }^{49,50}$.

337 Cell lines

338 HEK293T cells, HEK293T-hACE2 cells and Vero-E6-TMPRSS2 cells were maintained in 339 DMEM containing 10\% FBS, $2 \mathrm{mM}$ L-glutamine, $100 \mathrm{U} / \mathrm{mL} / \mathrm{mL}$ penicillin and incubated at $340 \quad 37 \square$ in a 5\% CO2 setting ${ }^{51}$. Expi293FTM cells were cultured in Expi293TM Expression 341 Medium (Thermo Fisher Scientific) at $37 \square$ in an incubator with $80 \%$ relative humidity and a $3425 \% \mathrm{CO} 2$ setting on an orbital shaker platform at $125 \pm 5 \mathrm{rpm} / \mathrm{min}$ (New Brunswick innova ${ }^{\mathrm{TM}}$ $3432100)$ according to the manufacturer's instructions.

\section{ELISA analysis of plasma and antibody binding to RBD and trimeric spike}

346 The recombinant RBD and trimeric spike proteins derived from SARS-CoV-2 (Sino 347 Biological) were diluted to final concentrations of $1 \mu \mathrm{g} / \mathrm{mL} / \mathrm{mL}$, then coated onto $96-$ well 348 plates (Corning 3690) and incubated at $4{ }^{\circ} \mathrm{C}$ overnight. Plates were washed with PBS-T (PBS 349 containing 0.05\% Tween-20) and blocked with blocking buffer (PBS containing 5\% skim 350 milk or $1 \% \mathrm{BSA}$ ) at $37^{\circ} \mathrm{C}$ for $1 \mathrm{~h}$. Serially diluted plasma samples or isolated monoclonal 
351 antibodies were added to the plates and incubated at $37^{\circ} \mathrm{C}$ for $1 \mathrm{~h}$. Wells were then incubated

352 with a secondary goat anti-human IgG labelled with horseradish peroxidase (HRP)

353 (Invitrogen) or with a rabbit polyclonal anti-human IgA alpha-chain labelled with HRP

354 (Abcam) and TMB substrate (SIGMA). Optical density (OD) at $450 \mathrm{~nm}$ was measured by a

355 spectrophotometer. Serially diluted plasma from healthy individuals or previously published

356 monoclonal antibodies against SARS-CoV-2 (B8) were used as negative controls.

Isolation of SARS-CoV-2 spike-specific IgG+ single memory B cells by FACS

359 RBD-specific single B cells were sorted as previously described ${ }^{52}$. In brief, PBMCs from 360 infected individuals were collected and incubated with an antibody cocktail and a His-tagged 361 RBD protein for identification of RBD-specific B cells. The cocktail consisted of the Zombie 362 viability dye (Biolegend), CD19-Percp-Cy5.5, CD3-Pacific Blue, CD14-Pacific Blue, CD56363 Pacific Blue, IgM-Pacific Blue, IgD-Pacific Blue, IgG-PE, CD27-PE-Cy7 (BD Biosciences) 364 and the recombinant SARS-CoV-2 Spike-His described above. Two consecutive staining 365 steps were conducted: the first one used an antibody and spike cocktail incubation of $30 \mathrm{~min}$ 366 at $4{ }^{\circ} \mathrm{C}$; the second staining involved staining with anti-His-APC and anti-His-FITC 367 antibodies (Abcam) at $4{ }^{\circ} \mathrm{C}$ for 30 min to detect the His tag of the RBD. The stained cells 368 were washed and resuspended in PBS containing 2\% FBS before being strained through a 70$369 \mu \mathrm{m}$ cell mesh filter (BD Biosciences). SARS-CoV-2 spike-specific single B cells were gated as CD19+CD27+CD3-CD14-CD56-IgM-IgD-IgG+Spike+ and sorted into 96-well PCR plates containing $10 \mu \mathrm{L}$ of RNAase-inhibiting RT-PCR catch buffer (1M Tris- $\mathrm{HCl} \mathrm{pH} 8.0$,

372 RNase inhibitor, DEPC-treated water). Plates were then snap-frozen on dry ice and stored at $373-80{ }^{\circ} \mathrm{C}$ until the reverse transcription reaction.

\section{Single B cell RT-PCR and antibody cloning}

376 Single memory B cells isolated from PBMCs of infected patients were cloned as previously 377 described ${ }^{53}$. Briefly, one-step RT-PCR was performed on sorted single memory B cell with a 378 gene specific primer mix, followed by nested PCR amplifications and sequencing using the 379 heavy chain and light chain specific primers. Cloning PCR was then performed using heavy 380 chain and light chain specific primers containing specific restriction enzyme cutting sites 381 (heavy chain, 5'-AgeI/3'-SalI; kappa chain, 5'-AgeI/3'-BsiWI). The PCR products were 382 purified and cloned into the backbone of antibody expression vectors containing the constant 383 regions of human $\operatorname{Ig} \gamma 1$. The constructed plasmids containing paired heavy and light chain 384 expression cassettes were co-transfected into 293T cells (ATCC) grown in 6-well plates. 
385 Antigen-specific ELISA and pseudovirus-based neutralization assays were used to analyze

386 the binding capacity to SARS-CoV-2 spike and the neutralization capacity of transfected

387 culture supernatants, respectively.

\section{Genetic analysis of the BCR repertoire}

390 Heavy chain and light chain germline assignment, framework region annotation, 391 determination of somatic hypermutation (SHM) levels (in nucleotides) and CDR loop lengths 392 (in amino acids) were performed with the aid of the NCBI/IgBlast tool suite 393 (https://www.ncbi.nlm.nih.gov/igblast/). Sequences were aligned using Clustal W in the 394 BioEdit sequence analysis package (Version 7.2). Antibody clonotypes were defined as a set of sequences that share genetic $\mathrm{V}$ and $\mathrm{J}$ regions as well as an identical CDR3.

\section{Antibody production and purification}

398 The paired antibody VH/VL chains were cloned into Ig $\gamma$ and $\operatorname{Ig} k$ expression vectors using T4 399 ligase (NEB). Antibodies produced from cell culture supernatants were purified immediately 400 by affinity chromatography using recombinant Protein G-Agarose (Thermo Fisher Scientific) 401 according to the manufacturer's instructions, to purify IgG. The purified antibodies were concentrated by an Amicon ultracentrifuge filter device (molecular weight cut-off $10 \mathrm{kDa}$; Millipore) to a volume of $0.2 \mathrm{~mL}$ in PBS (Life Technologies), and then stored at $4{ }^{\circ} \mathrm{C}$ or $80{ }^{\circ} \mathrm{C}$ for further characterization.

\section{Pseudovirus-based neutralization assay}

407 The neutralizing activity of NAbs was determined using a pseudotype-based neutralization 408 assay as we previously described ${ }^{54}$. Briefly, The pseudovirus was generated by co409 transfection of 293T cells with pVax-1-S-COVID19 and pNL4-3Luc_Env_Vpr, carrying the 410 optimized spike (S) gene (QHR63250) and a human immunodeficiency virus type 1 411 backbone, respectively ${ }^{54}$. Viral supernatant was collected at $48 \mathrm{~h}$ post-transfection and frozen 412 at $-80{ }^{\circ} \mathrm{C}$ until use. The serially diluted monoclonal antibodies or sera were incubated with 413200 TCID50 of pseudovirus at $37{ }^{\circ} \mathrm{C}$ for 1 hour. The antibody-virus mixtures were 414 subsequently added to pre-seeded HEK 293T-ACE2 cells. 48 hours later, infected cells were 415 lysed to measure luciferase activity using a commercial kit (Promega, Madison, WI). Half416 maximal (IC50) or 90\% (IC90) inhibitory concentrations of the evaluated antibody were 417 determined by inhibitor vs. normalized response -- 4 Variable slope using GraphPad Prism 8 418 or later (GraphPad Software Inc.). 
420 Neutralization activity of monoclonal antibodies against authentic SARS-CoV-2

421 The SARS-CoV-2 focus reduction neutralization test (FRNT) was performed in a certified

422 Biosafety level 3 laboratory. Neutralization assays against live SARS-CoV-2 were conducted

423 using a clinical isolate previously obtained from a nasopharyngeal swab from an infected

424 patient ${ }^{55}$. The tested antibodies were serially diluted, mixed with $50 \mu \mathrm{L}$ of SARS-CoV-2

$425\left(1 \times 10^{3}\right.$ focus forming unit $\left./ \mathrm{mL}, \mathrm{FFU} / \mathrm{mL}\right)$ in 96 -well plates, and incubated for 1 hour at $37^{\circ} \mathrm{C}$.

426 Mixtures were then transferred to 96 -well plates pre-seeded with $1 \times 10^{4} /$ well Vero E6 cells

427 and incubated at $37^{\circ} \mathrm{C}$ for 24 hours. The culture medium was then removed, and the plates

428 were air-dried in a biosafety cabinet (BSC) for 20 mins. Cells were then fixed with a $4 \%$

429 paraformaldehyde solution for $30 \mathrm{~min}$ and air-dried in the BSC again. Cells were further

430 permeabilized with $0.2 \%$ Triton $\mathrm{X}-100$ and incubated with cross-reactive rabbit sera anti-

431 SARS-CoV-2-N for 1 hour at RT before adding an Alexa Fluor 488 goat anti-rabbit IgG

$432(\mathrm{H}+\mathrm{L})$ cross-adsorbed secondary antibody (Life Technologies). The fluorescence density of

433 SARS-CoV-2 infected cells were scanned using a Sapphire Biomolecular Imager (Azure

434 Biosystems) and the neutralization effects were then quantified using Fiji software (NIH).

436 Antibody binding kinetics and competition between antibodies measured by Surface

437 Plamon Resonance (SPR)

438 The binding kinetics and affinity of recombinant monoclonal antibodies for the SARS-CoV-2

439 RBD protein (SinoBiological) were analyzed by SPR (Biacore T200, GE Healthcare).

440 Specifically, the SARS-CoV-2 RBD protein was covalently immobilized to a CM5 sensor

441 chip via amine groups in 10mM sodium acetate buffer $(\mathrm{pH} 5.0)$ for a final RU around 250.

442 SPR assays were run at a flow rate of $10 \mathrm{uL} / \mathrm{min}$ in HEPES buffer. For conventional

443 kinetic/dose-response, serial dilutions of monoclonal antibodies were injected across the

444 spike protein surface for 180 s, followed by a 900 s dissociation phase using a multi-cycle

445 method. Remaining analytes were removed in the surface regeneration step with the injection

446 of $10 \mathrm{mM}$ glycine- $\mathrm{HCl}(\mathrm{pH} 1.5)$ for $60 \mathrm{~s}$ at a flow rate of $30 \mu \mathrm{l} / \mathrm{min}$. Kinetic analysis of each

447 reference subtracted injection series was performed using the Biacore Insight Evaluation

448 Software (GE Healthcare). All sensorgram series were fit to a 1:1 (Langmuir) binding model

449 of interaction. Before evaluating the competition between antibodies, both the saturating

450 binding concentrations of antibodies for the immobilized SARS-CoV-2 RBD protein were

451 determined separately. In the competitive assay, antibodies at the saturating concentration

452 were injected onto the chip with immobilized spike protein for 120 s until binding steady-state 
453 was reached. The other antibody also used at the saturating concentration was then injected

454 for 120 s, followed by another 120 s of injection of antibody to ensure a saturation of the

455 binding reaction against the immobilized RBD protein. The differences in response units

456 between antibody injection alone and prior antibody incubation reflect the antibodies'

457 competitive ability by binding to the RBD protein.

\section{Model building of ZCB11 and structure presentation}

460 A model of ZCB11 variable regions was generated based on the protein sequence by the

461 SWISS-MODEL using the crystal structure of S2E12 Fab fragment (Research Collaboratory

462 for Structural Bioinformatics [RCSB] PDB code 7K3Q) as the template. The structure

463 alignment, cartoon representations, labeling of amino acids in RBD (from PDB 7K45) were

464 generated by PyMOL.

465

\section{Hamster experiments}

467 In vivo evaluation of monoclonal antibody ZB8 or ZCB11 in the established golden Syrian

468 hamster model of SARS-CoV-2 infection was performed as described previously, with slight 469 modifications ${ }^{33}$. The animal experiments were approved by the Committee on the Use of 470 Live Animals in Teaching and Research (CULATR 5359-20) of the University of Hong 471 Kong (HKU). Briefly, 6-10-week-old male and female hamsters were obtained from the 472 Chinese University of Hong Kong Laboratory Animal Service Centre through the HKU 473 Centre for Comparative Medicine Research. The hamsters were housed with access to 474 standard pellet feed and water ad libitum until live virus challenge in the BSL-3 animal 475 facility at Department of Microbiology, HKU. The viral challenge experiments were then 476 conducted in our Biosafety Level-3 animal facility following SOPs strictly, with strict 477 adherence to SOPs. The hamsters were randomized from different litters into experimental 478 groups. Experiments were performed in compliance with the relevant ethical regulations ${ }^{33}$. 479 For prophylaxis studies, 24 hours before live virus challenge, three groups of hamsters were 480 intraperitoneally administered with one dose of test antibody in phosphate-buffered saline 481 (PBS) at the indicated dose. At day 0, each hamster was intranasally inoculated with a 482 challenge dose of $100 \mu \mathrm{L}$ of Dulbecco's Modified Eagle Medium containing $10^{5}$ PFU of 483 SARS-CoV-2 Delta variant or Omicron variant under anesthesia with intraperitoneal 484 ketamine $(200 \mathrm{mg} / \mathrm{kg})$ and xylazine $(10 \mathrm{mg} / \mathrm{kg})$. The hamsters were monitored daily for 485 clinical signs of disease. Syrian hamsters typically clear virus within one week after SARS486 CoV-2 infection. Accordingly, animals were sacrificed for analysis at day 4 after virus 
487 challenge with high viral loads ${ }^{33}$. Half the nasal turbinate, trachea, and lung tissues were

488 used for viral load determination by quantitative RT-qPCR assay ${ }^{56}$ and infectious virus

489 titration by plaque assay ${ }^{33}$ as we described previously.

490

491 Quantification and statistical analysis

492 Statistical analysis was performed using PRISM 8.0 or later. Ordinary one-way ANOVA and

493 multiple comparisons were used to compare group means and differences between multiple

494 groups. Unpaired Student's t tests were used to compare group means between two groups

495 only. A P-value <0.05 was considered significant. The number of independent experiments

496 performed, the number of animals in each group, and the specific details of statistical tests are reported in the figure legends and the Methods section.

\section{SUPPLEMENTAL INFORMATION}

500 The supplemental information includes 7 Tables and 3 Figures.

501

502 ACKNOWLEDGMENTS

503 This study was supported by the Hong Kong Research Grants Council Collaborative 504 Research Fund (C7156-20G, C1134-20G and C5110-20G) and Shenzhen Science and 505 Technology Program (JSGG20200225151410198 and JCYJ20210324131610027); the Hong 506 Kong Health@InnoHK, Innovation and Technology Commission; and the China National 507 Program on Key Research Project (2020YFC0860600, 2020YFA0707500 and 508 2020YFA0707504); and donations from the Friends of Hope Education Fund. Z.C.'s team 509 was also partly supported by the Hong Kong Theme-Based Research Scheme (T11-706/18$510 \mathrm{~N})$. This study was also partly supported by funding the Health and Medical Research Fund, 511 the Food and Health Bureau, The Government of the Hong Kong Special Administrative 512 Region (Ref no.: COVID1903010-Project 7 and 20190572); the Consultancy Service for

513 Enhancing Laboratory Surveillance of Emerging Infectious Diseases and Research Capability 514 on Antimicrobial Resistance for Department of Health of the Hong Kong Special 515 Administrative Region Government; the National Program on Key Research Project of China 516 (grant no. 2020YFA0707500 and 2020YFA0707504); Sanming Project of Medicine in 517 Shenzhen, China (grant no. SZSM201911014); the High Level-Hospital Program, Health 518 Commission of Guangdong Province, China; the Major Science and Technology Program of 519 Hainan Province (ZDKJ202003); and the research project of Hainan academician innovation 520 platform (YSPTZX202004); and donations from the Shaw Foundation of Hong Kong, the 
521 Richard Yu and Carol Yu, Michael Seak-Kan Tong, May Tam Mak Mei Yin, Lee Wan

522 Keung Charity Foundation Limited, the Providence Foundation Limited (in memory of the 523 late Lui Hac Minh), Hong Kong Sanatorium \& Hospital, Hui Ming, Hui Hoy and Chow Sin 524 Lan Charity Fund Limited, Chan Yin Chuen Memorial Charitable Foundation, Marina Man525 Wai Lee, the Hong Kong Hainan Commercial Association South China Microbiology 526 Research Fund, the Jessie \& George Ho Charitable Foundation, Perfect Shape Medical 527 Limited, Kai Chong Tong, Tse Kam Ming Laurence, Foo Oi Foundation Limited, Betty 528 Hing-Chu Lee, Ping Cham So, and Lo Ying Shek Chi Wai Foundation. The funding sources 529 had no role in the study design, data collection, analysis, interpretation, or writing of the 530 report. Finally, we thank Dr. David D. Ho for kindly providing the expression plasmids 531 encoding for D614G, B.1.1.7 and B.1.351 variants and Dr. Linqi Zhang for B.1.617.2.

\section{AUTHOR CONTRIBUTIONS}

534 Conceptualization, Z.C.; HuNAb cloning, Z.B.; experimental design, Z.C., Z.B., R.Z., J.F.535 W.C.; hamster experiments, J.F.-W.C., V.K.-M.P., C.C.-S.C., J.O.-L.T., , C.C.-Y.C.; clinical 536 specimens, Q.P., K.K.-W.T.; pseudovirus neutralization assay, M.L., Q.P., B.C.; authentic 537 virus neutralizing assay, R.Z., B.W.-Y.M., P.W., H.C., L.L., H.C.; viral plaque assay, S.Y.; 538 plasmid cloning, M.L., B.C., H.-O.M. viral RNA measurement, K.-K.A.; critical comments, 539 supports and materials, K.-Y.Y.

541 DECLARATION OF INTEREST

542 J.F.-W.C. has received travel grants from Pfizer Corporation Hong Kong and Astellas 543 Pharma Hong Kong Corporation Limited and was an invited speaker for Gilead Sciences 544 Hong Kong Limited and Luminex Corporation. The funding sources had no role in study 545 design, data collection, analysis or interpretation or writing of the report. The other authors 546 declare no conflicts of interest except for a provisional patent application filed for human 547 monoclonal antibodies generated in our laboratory. Z.C., B.Z., and R.Z. are the co-inventers 548 of NAbs ZCB11, ZCB3 and ZCD3.

\section{Reporting Summary}

551 Further information on research design is available in the Nature Research Reporting 552 Summary linked to this article. 
555 The data of this studies are available upon reasonable request and accession codes will be

556 available before publication.

557

558 Code availability

559 No custom computer code or algorithm used to generate results that are reported in the paper

560 and central to its main claims.

561

562 REFERENCES

5631 Polack, F. P. et al. Safety and Efficacy of the BNT162b2 mRNA Covid-19 Vaccine. $N$

$564 \quad$ Engl J Med 383, 2603-2615, doi:10.1056/NEJMoa2034577 (2020).

5652 Baden, L. R. et al. Efficacy and Safety of the mRNA-1273 SARS-CoV-2 Vaccine. $N$

$566 \quad$ Engl J Med 384, 403-416, doi:10.1056/NEJMoa2035389 (2021).

5673 Voysey, M. et al. Safety and efficacy of the ChAdOx1 nCoV-19 vaccine (AZD1222)

568 against SARS-CoV-2: an interim analysis of four randomised controlled trials in

569 Brazil, South Africa, and the UK. Lancet 397, 99-111, doi:10.1016/S0140-

570 6736(20)32661-1 (2021).

5714 Tanriover, M. D. et al. Efficacy and safety of an inactivated whole-virion SARS-

$572 \mathrm{CoV}-2$ vaccine (CoronaVac): interim results of a double-blind, randomised, placebo-

573 controlled, phase 3 trial in Turkey. Lancet 398, 213-222, doi:10.1016/S0140-

574 6736(21)01429-X (2021).

5755 Xia, S. et al. Safety and immunogenicity of an inactivated SARS-CoV-2 vaccine,

576 BBIBP-CorV: a randomised, double-blind, placebo-controlled, phase 1/2 trial. Lancet

577

578

579

580

581

582

583

584

585

586

587

588

589

590

591

592

593

594

595

596

597 Infect Dis 21, 39-51, doi:10.1016/S1473-3099(20)30831-8 (2021).

6 Birhane, M. et al. COVID-19 Vaccine Breakthrough Infections Reported to CDC United States, January 1-April 30, 2021. MMWR Morb Mortal Wkly Rep 70, 792-793, doi:http://dx.doi.org/10.15585/mmwr.mm7021e3 (2021).

7 Abu-Raddad, L. J., Chemaitelly, H. \& Bertollini, R. Severity of SARS-CoV-2 Reinfections as Compared with Primary Infections. New Engl J Med, doi:10.1056/NEJMc2108120 (2021).

8 To, K. K. et al. Coronavirus Disease 2019 (COVID-19) Re-infection by a Phylogenetically Distinct Severe Acute Respiratory Syndrome Coronavirus 2 Strain Confirmed by Whole Genome Sequencing. Clin Infect Dis 73, e2946-e2951, doi:10.1093/cid/ciaa1275 (2021).

9 Khan, N. A., Al-Thani, H. \& El-Menyar, A. The emergence of new SARS-CoV-2 variant (Omicron) and increasing calls for COVID-19 vaccine boosters-The debate continues. Travel Med Infect Dis 45, 102246, doi:10.1016/j.tmaid.2021.102246 (2021).

10 Tao, K. et al. The biological and clinical significance of emerging SARS-CoV-2 variants. Nat Rev Genet 22, 757-773, doi:10.1038/s41576-021-00408-x (2021).

11 Wang, Y. et al. Transmission, viral kinetics and clinical characteristics of the emergent SARS-CoV-2 Delta VOC in Guangzhou, China. EClinicalMedicine 40, 101129, doi:10.1016/j.eclinm.2021.101129 (2021).

598

599

Zhang, Q. et al. Potent and protective IGHV3-53/3-66 public antibodies and their shared escape mutant on the spike of SARS-CoV-2. Nat Commun 12, 4210, doi:10.1038/s41467-021-24514-w (2021). 
60013 Qiaoli Peng, R. Z., Yuewen Wang, Meiqing Zhao, Na Liu, Shuang Li, Haode Huang,

601

602

603

604

605

606

607

608

609

610

611

612

613

614

615

616

617

618

619

620

621

622

623

624

625

626

627

628

629

630

631

632

633

634

635

636

637

638

639

640

641

642

643

644

645

646

647

648

649

Dawei Yang, Ka-Kit Au, Hui Wang, Kwan Man, Kwok-Yung Yuen, Zhiwei Chen. Waning immune responses against SARS-CoV-2 among vaccinees in Hong Kong. BioRxiv, doi:https://doi.org/10.1101/2021.12.22.473934 (2021).

14 Frederic Grabowski, M. K., Tomasz Lipniacki. Omicron strain spreads with the doubling time of 3.2-3.6 days in South Africa province of Gauteng that achieved herd immunity to Delta variant. medRxiv, doi:https://doi.org/10.1101/2021.12.08.21267494 (2021).

15 WHO. Enhancing Readiness for Omicron (B.1.1.529): Technical Brief and Priority Actions for Member States. Overview (2021).

16 Pulliam, J. R. C. et al. Increased risk of SARS-CoV-2 reinfection associated with emergence of the Omicron variant in South Africa. medRxiv, 2021.2011.2011.21266068, doi:10.1101/2021.11.11.21266068 (2021).

17 Espenhain, L. et al. Epidemiological characterisation of the first 785 SARS-CoV-2 Omicron variant cases in Denmark, December 2021. Euro Surveill 26, doi:10.2807/1560-7917.ES.2021.26.50.2101146 (2021).

18 Planas, D. et al. Reduced sensitivity of SARS-CoV-2 variant Delta to antibody neutralization. Nature 596, 276-280, doi:10.1038/s41586-021-03777-9 (2021).

19 Zhou, R. et al. Vaccine-breakthrough infection by the SARS-CoV-2 Omicron variant elicits broadly cross-reactive immune responses. bioRxiv, 2021.2012.2027.474218, doi:10.1101/2021.12.27.474218 (2021).

20 Planas, D., Saunders, N. \& Schwartz, O. Considerable escape of SARS-CoV-2 Omicron to antibody neutralization. Nature https://doi.org/10.1038/d41586-02103827-2 (2021).

21 Liu, L., Iketani, S. \& Ho, D. D. Striking antibody evasion manifested by the Omicron variant of SARS-CoV-2. Nature https://doi.org/10.1038/d41586-021-03826-3 (2021).

22 Cele, S., Jackson, L. \& Sigal, A. Omicron extensively but incompletely escapes Pfizer BNT162b2 neutralization. Nature https://doi.org/10.1038/d41586-021-03824-5 (2021).

23 Cameroni, E., Bown, J. \& Corti, D. Broadly neutralizing antibodies overcome SARSCoV-2 Omicron antigenic shift. Nature https://doi.org/10.1038/d41586-021-03825-4 (2021).

24 Lu, L. et al. Neutralization of SARS-CoV-2 Omicron variant by sera from BNT162b2 or Coronavac vaccine recipients. Clin Infect Dis, doi:10.1093/cid/ciab1041 (2021).

25 Baisheng Li, A. D., Kuibiao Li, Yao Hu, Zhencui Li, Qianling Xiong, Zhe Liu, Qianfang Guo, Lirong Zou, Huan Zhang, Meng Zhang, Fangzhu Ouyang, Juan Su, Wenzhe Su, Jing Xu, Huifang Lin, Jing Sun, Jinju Peng, Huiming Jiang, Pingping Zhou, Ting Hu, Min Luo, Yingtao Zhang, Huanying Zheng, Jianpeng Xiao, Tao Liu, Rongfei Che, Hanri Zeng, Zhonghua Zheng, Yushi Huang, Jianxiang Yu, Lina Yi, Jie Wu, Jingdiao Chen, Haojie Zhong, Xiaoling Deng, Min Kang, Oliver G. Pybus, Matthew Hall, Katrina A. Lythgoe, Yan Li, Jun Yuan, Jianfeng He, Jing Lu. Viral infection and transmission in a large, well-traced outbreak caused by the SARS-CoV2 Delta variant. MeoRxiv, doi:https://doi.org/10.1101/2021.07.07.21260122 (2021).

26 Wang, G. L. et al. Susceptibility of Circulating SARS-CoV-2 Variants to Neutralization. N Engl J Med 384, 2354-2356, doi:10.1056/NEJMc2103022 (2021).

27 Garcia-Beltran, W. F. et al. COVID-19-neutralizing antibodies predict disease severity and survival. Cell 184, 476-488 e411, doi:10.1016/j.cell.2020.12.015 (2021).

28 Biao Zhou, R. Z., Jasper Fuk-Woo Chan, Jianwei Zeng, Qi Zhang, Shuofeng Yuan, Li Liu, Rémy Robinot, Sisi Shan, Jiwan Ge, Hugo Yat-Hei Kwong, Dongyan Zhou, Haoran Xu, Chris Chan, Vincent Poon, Hin Chu, Ming Yue, Ka-Yi Kwan, Chun Yin 
650

651

652

653

654

655

656

657

658

659

660

661

662

663

664

665

666

667

668

669

670

671

672

673

674

675

676

677

678

679

680

681

682

683

684

685

686

687

688

689

690

691

692

693

694

695

696

697

698

699

Chan, Na Liu, Chris Chun-Yiu Chan, Kenn Ka-Heng Chik, Zhenglong Du, Ka-Kit Au, Haode Huang, Hiu-On Man, Jianli Cao, Cun Li, Ziyi Wang, Jie Zhou, Youqiang Song, Man Lung Yeung, Kelvin To, David Ho, Lisa A. Chakrabarti, Xinquan Wang, Linqi Zhang, Kwok-Yung Yuen, Zhiwei Chen. SARS-CoV-2 hijacks neutralizing dimeric IgA for enhanced nasal infection and injury. BioRxiv, doi:https://doi.org/10.1101/2021.10.05.463282 (2021).

29 Schmitz, A. J. et al. A vaccine-induced public antibody protects against SARS-CoV-2 and emerging variants. Immunity 54, 2159-2166 e2156, doi:10.1016/j.immuni.2021.08.013 (2021).

30 Tan, T. J. C. et al. Sequence signatures of two public antibody clonotypes that bind SARS-CoV-2 receptor binding domain. Nat Commun 12, 3815, doi:10.1038/s41467021-24123-7 (2021).

31 Yunlong Cao, J. W., Fanchong Jian, Tianhe Xiao, Weiliang Song, Ayijiang Yisimayi, Weijin Huang, Qianqian Li, Peng Wang, Ran An, Jing Wang, Yao Wang, Xiao Niu, Sijie Yang, Hui Liang, Haiyan Sun, Tao Li, Yuanling Yu, Qianqian Cui, Shuo Liu, Xiaodong Yang, Shuo Du, Zhiying Zhang, Xiaohua Hao, Fei Shao, Ronghua Jin, Xiangxi Wang, Junyu Xiao, Youchun Wang, Xiaoliang Sunney Xie. Omicron escapes the majority of existing SARS-CoV-2 neutralizing antibodies. BioRxiv, doi:https://doi.org/10.1101/2021.12.07.470392 (2021).

32 Tortorici, M. A. et al. Ultrapotent human antibodies protect against SARS-CoV-2 challenge via multiple mechanisms. Science 370, 950-957, doi:10.1126/science.abe3354 (2020).

33 Chan, J. F.-W. et al. Simulation of the clinical and pathological manifestations of Coronavirus Disease 2019 (COVID-19) in golden Syrian hamster model: implications for disease pathogenesis and transmissibility. Clinical Infectious Diseases 71, 24282446, doi:10.1093/cid/ciaa325 (2020).

34 Zhou, D. et al. Robust SARS-CoV-2 infection in nasal turbinates after treatment with systemic neutralizing antibodies. Cell Host Microbe 29, 551-563 e555, doi:10.1016/j.chom.2021.02.019 (2021).

35 Yang, T. J. et al. Effect of SARS-CoV-2 B.1.1.7 mutations on spike protein structure and function. Nat Struct Mol Biol 28, 731-739, doi:10.1038/s41594-021-00652-z (2021).

36 Dejnirattisai, W. et al. Antibody evasion by the P.1 strain of SARS-CoV-2. Cell 184, 2939-2954 e2939, doi:10.1016/j.cell.2021.03.055 (2021).

37 Hoffmann, M. et al. SARS-CoV-2 variants B.1.351 and P.1 escape from neutralizing antibodies. Cell 184, 2384-2393 e2312, doi:10.1016/j.cell.2021.03.036 (2021).

38 Wang, P. et al. Increased resistance of SARS-CoV-2 variant P.1 to antibody neutralization. Cell Host Microbe 29, 747-751 e744, doi:10.1016/j.chom.2021.04.007 (2021).

39 Li, M., Lou, F. \& Fan, H. SARS-CoV-2 Variants of Concern Delta: a great challenge to prevention and control of COVID-19. Signal Transduct Target Ther 6, 349, doi:10.1038/s41392-021-00767-1 (2021).

40 Liu, C. et al. Reduced neutralization of SARS-CoV-2 B.1.617 by vaccine and convalescent serum. Cell 184, 4220-4236 e4213, doi:10.1016/j.cell.2021.06.020 (2021).

41 Mlcochova, P. et al. SARS-CoV-2 B.1.617.2 Delta variant replication and immune evasion. Nature 599, 114-119, doi:10.1038/s41586-021-03944-y (2021).

42 Karim, S. S. A. \& Karim, Q. A. Omicron SARS-CoV-2 variant: a new chapter in the COVID-19 pandemic. Lancet 398, 2126-2128, doi:10.1016/S0140-6736(21)02758-6 (2021). 
700

701

702

703

704

705

706

707

708

709

710

711

712

713

714

715

716

717

718

719

720

721

722

723

724

725

726

727

728

729

730

731

732

733

734

735

736

737

738

739

740

741

742

743

744

745

746

43 Dejnirattisai, W. et al. Reduced neutralisation of SARS-CoV-2 omicron B.1.1.529 variant by post-immunisation serum. Lancet, doi:10.1016/S0140-6736(21)02844-0 (2021).

44 Chen, R. E. et al. In vivo monoclonal antibody efficacy against SARS-CoV-2 variant strains. Nature 596, 103-108, doi:10.1038/s41586-021-03720-y (2021).

45 Barnes, C. O. et al. SARS-CoV-2 neutralizing antibody structures inform therapeutic strategies. Nature 588, 682-687, doi:10.1038/s41586-020-2852-1 (2020).

46 Yuan, M. et al. Structural and functional ramifications of antigenic drift in recent SARS-CoV-2 variants. bioRxiv, doi:10.1101/2021.02.16.430500 (2021).

47 Dong, J. et al. Genetic and structural basis for recognition of SARS-CoV-2 spike protein by a two-antibody cocktail. bioRxiv, doi:10.1101/2021.01.27.428529 (2021).

48 Wang, L. et al. Ultrapotent antibodies against diverse and highly transmissible SARSCoV-2 variants. Science 373, doi:10.1126/science.abh1766 (2021).

49 Chan, J. F. et al. Low Environmental Temperature Exacerbates Severe Acute Respiratory Syndrome Coronavirus 2 Infection in Golden Syrian Hamsters. Clin Infect Dis, doi:10.1093/cid/ciab817 (2021).

50 Chan, J. F. et al. Surgical Mask Partition Reduces the Risk of Noncontact Transmission in a Golden Syrian Hamster Model for Coronavirus Disease 2019 (COVID-19). Clin Infect Dis 71, 2139-2149, doi:10.1093/cid/ciaa644 (2020).

51 Liu, L. et al. Anti-spike IgG causes severe acute lung injury by skewing macrophage responses during acute SARS-CoV infection. JCI Insight 4, e123158, doi:10.1172/jci.insight.123158 (2019).

52 Kong, L. et al. Key gp120 Glycans Pose Roadblocks to the Rapid Development of VRC01-Class Antibodies in an HIV-1-Infected Chinese Donor. Immunity 44, 939-950, doi:10.1016/j.immuni.2016.03.006 (2016).

53 Smith, K. et al. Rapid generation of fully human monoclonal antibodies specific to a vaccinating antigen. Nat Protoc 4, 372-384, doi:10.1038/nprot.2009.3 (2009).

54 Poeran, J., Zhong, H., Wilson, L., Liu, J. \& Memtsoudis, S. G. Cancellation of elective surgery and intensive care unit capacity in New York state: a retrospective cohort analysis. Anesth Analg 131, 1337-1341, doi:10.1213/ANE.0000000000005083 (2020).

55 Chu, H. et al. Comparative tropism, replication kinetics, and cell damage profiling of SARS-CoV-2 and SARS-CoV with implications for clinical manifestations, transmissibility, and laboratory studies of COVID-19: an observational study. The Lancet Microbe 1, e14-e23, doi:10.1016/S2666-5247(20)30004-5 (2020).

56 Chan, J. F. et al. Improved Molecular Diagnosis of COVID-19 by the Novel, Highly Sensitive and Specific COVID-19-RdRp/Hel Real-Time Reverse Transcription-PCR Assay Validated In Vitro and with Clinical Specimens. J Clin Microbiol 58, e0031000320, doi:10.1128/JCM.00310-20 (2020).

\section{FIGURE LEGENDS}

Fig. 1. Identification of an elite vaccinee who developed bNAbs. Plasma samples derived from 34 BNT162b2-vaccinees were tested at average 30.7 days (range 7-47 days) after second vaccination (BioNTech-Pfizer). (a) Serially diluted plasma samples were subjected to neutralization assay against the pseudotyped SARS-CoV-2 WT and five variants of concern. The neutralizing curve of the elite BNT162b2-26 vaccinee (red) was compared with the mean 
747

748

749

750

751

752

753

754

755

756

757

758

759

760

761

762

763

764

765

766

767

768

769

770

771

772

773

774

775

776

777

778

779

780

curve of all vaccinees tested (dark black). (b) Binding activity of spike-specific plasma IgG was determined by ELISA at serial dilutions. The binding curve of the elite BNT162b2-26 vaccinee was presented as red. (c) The neutralization antibody potency index was defined by the ratio of $\mathrm{IC}_{50} / \mathrm{AUC}$ of anti-spike $\mathrm{IgG}$ in BNT162b2-vaccinees. Neutralizing $\mathrm{IC}_{50}$ values represented plasma dilution required to achieve $50 \%$ virus neutralization. The area under the curve (AUC) represented the total peak area was calculated from ELISA OD values. Each symbol represented an individual vaccinee with a line indicating the median of each group. The elite BNT162b2-26 vaccinee was presented as red dots.

Fig. 2. Comparison of bNAbs isolated from the elite vaccinee. (a) RBD- and spike-specific binding activities of 4 newly cloned NAbs including ZCB3, ZCB11, ZCC10 and ZCD3 were determined by ELISA at serial dilutions. A known NAb ZB8 was included as a control. (b) Neutralizing activities of ZCB3, ZCB11, ZCC10 and ZCD3 were determined against six pseudotyped SARS-CoV-2 variants of concern including D614G (WT), Alpha, Beta, Gamma, Delta and Omicron as compared with the control NAb ZB8. (c) Neutralizing activities of ZCB3, ZCB11, ZCC10 and ZCD3 were determined against the same six but authentic SARSCoV-2 variants of concern as compared with the control NAb ZB8. The color coding was consistently used in a-c. Notably, the authentic SARS-CoV-2 Omicron ${ }_{\text {R346K }}$ was also tested (bottom right in c with empty dots). The dashed line in each graph indicated 50\% neutralization.

\section{Fig. 3. Naturally occurring mutations or deletions conferring antibody resistance in} VOCs. (a) Fold change of $\mathrm{IC}_{50}$ values relative to WT was determined by pseudoviruses carrying individual mutations or deletion against bNAbs ZCB3 and ZCB11 as compared with ZB8. (b) Antibody competition by SPR between ZCB11 and ZC8 (top) as well as between ZCB11 and ZCB3 (bottom). (c) Structural alignment between S2E12 and ZCB11 variable regions. The structure of the ZCB11 variable region predicted by the SWISS-MODEL is superimposed into the structure of S2E12 (PDB: 7K3Q). Cartoon representation of ZCB11 variable region of heavy chain $(\mathrm{VH})$ is shown in purple and the variable region of light chain (VK) in orange. The S2E12 VH and VK are shown in yellow and green, respectively. The CDRs of VH and VK are labelled. (d) The structure of RBD in complex with the S2E12 variable region (from PDB 7K45). RBD is shown in cyan with receptor binding motif (RBM) highlighted in light pink and the amino acids whose substitution confers resistance to ZCB11 in (a) are highlighted in red. 
782 Fig. 4. Efficacy of ZCB11 against authentic SARS-CoV-2 Delta and Omicron in golden

783 Syrian hamsters as compared with ZB8. (a) Experimental schedule and color coding for

784 different treatment groups. Three groups of hamsters $(n=8)$ received a single intraperitoneal

785 injection of PBS (grey), $4.5 \mathrm{mg} / \mathrm{kg}$ of ZB8 (purple) or $4.5 \mathrm{mg} / \mathrm{kg}$ of ZCB11 (blue) at one day

786 before viral infection (-1 dpi). 24 hours later (day 0), each group was divided into two

787 subgroups for intranasal challenge with $10^{5}$ PFU live SARS-CoV-2 Delta and Omicron

788 variants, respectively. All animals were sacrificed on day 4 for final analysis. (b, e) Daily

789 body weight was measured after viral infection. (c, f) The nucleocapsid protein (NP)

790 subgenomic RNA copy numbers (normalized by $\beta$-actin) in lung homogenates were

791 determined by a sensitive RT PCR. (d, g) Live viral plaque assay was used to quantify the

792 number of infectious viruses in lung homogenates. Log10-transformed plaque-forming units

793 (PFU) per mL were shown for each group. LOD: limit of detection. Each symbol represents

794 an individual hamster with a line indicating the mean of each group. The color coding was

795 consistently used in each graph. Statistics were generated using one-way ANOVA followed

796 by Tukey's multiple comparisons test. **p<0.01;***p<0.001. 
Fig. 1

a

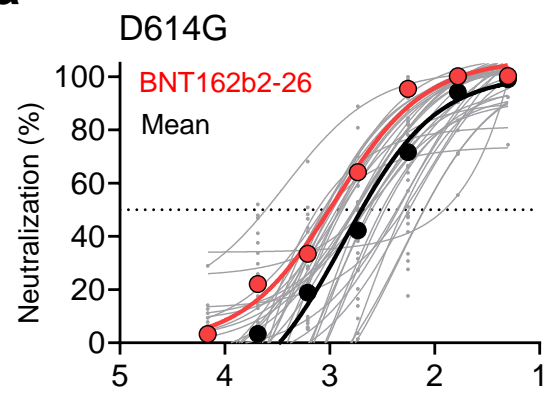

Beta

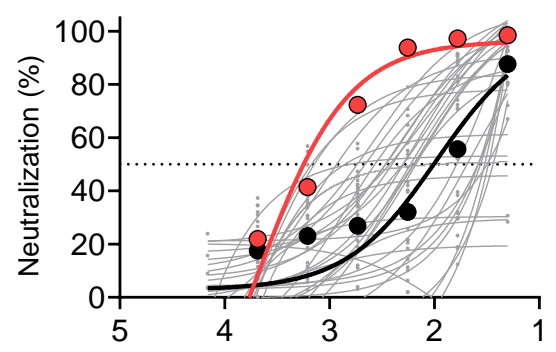

Delta

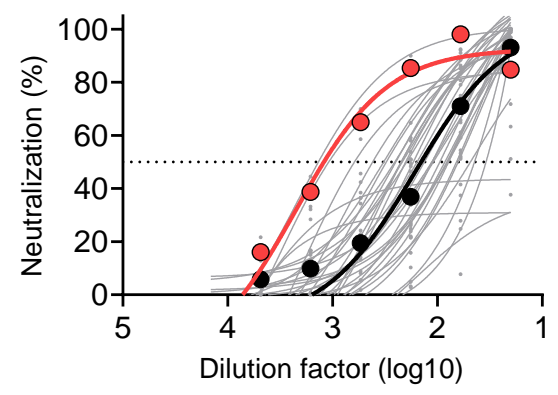

b

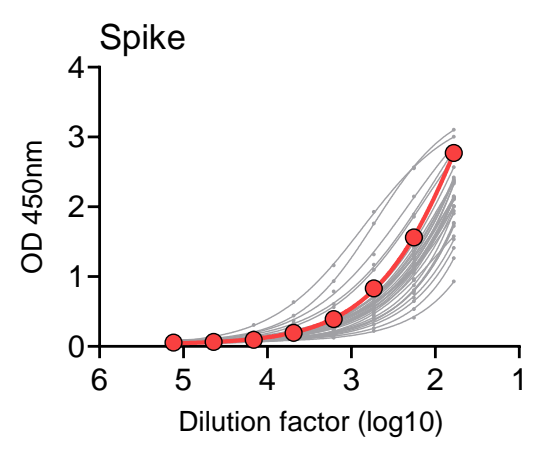

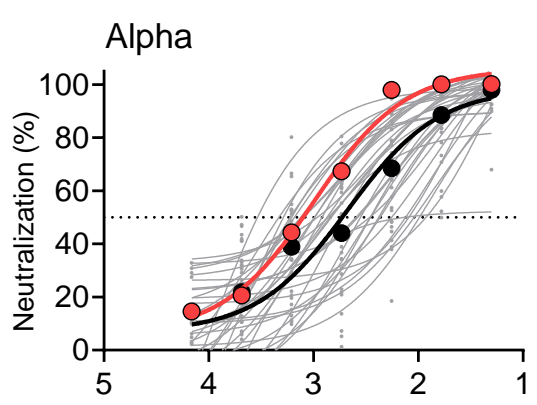

Gamma

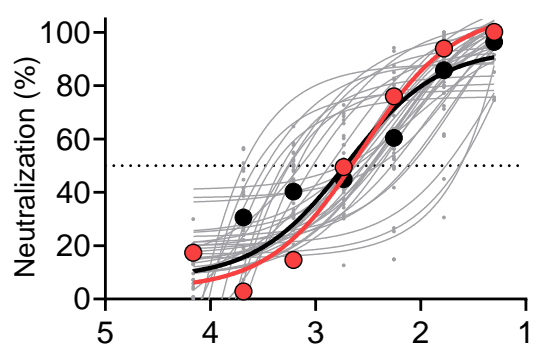

Omicron

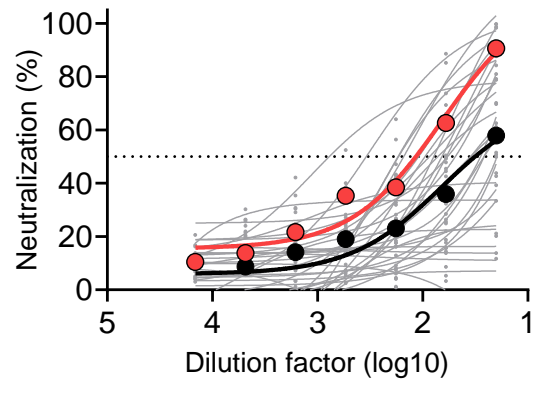

C

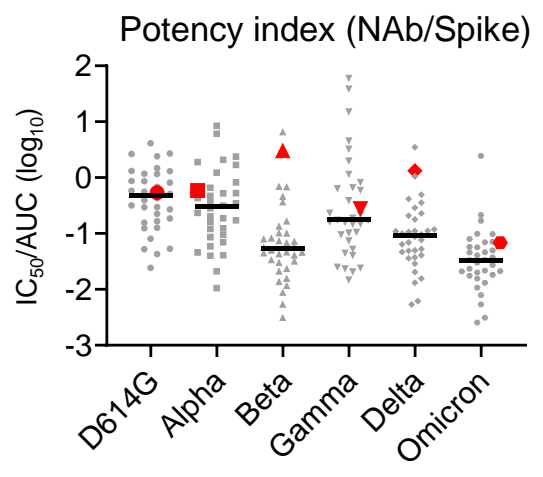



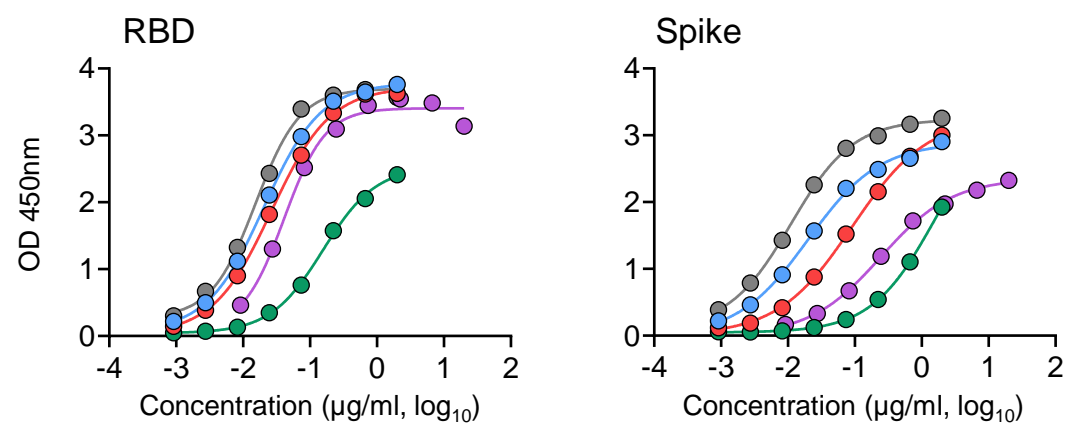

\section{ZCB3 \\ ZCB11 \\ ZCC10 \\ ZCD3 \\ ZB8}

b

\section{Pseudovirus}

D614G

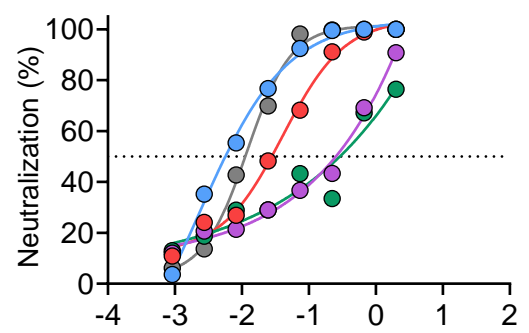

Gamma

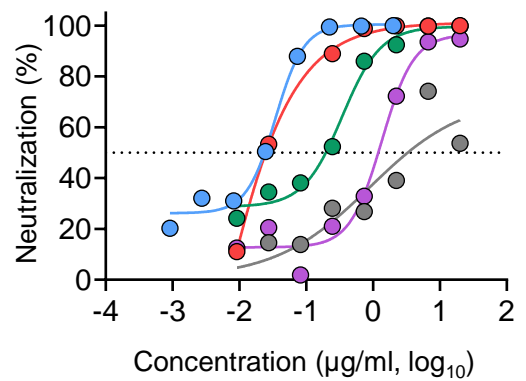

C

D614G

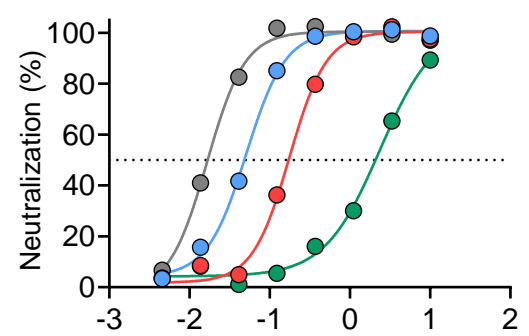

Gamma

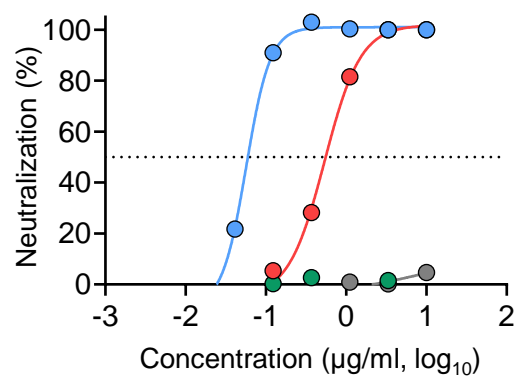

Alpha

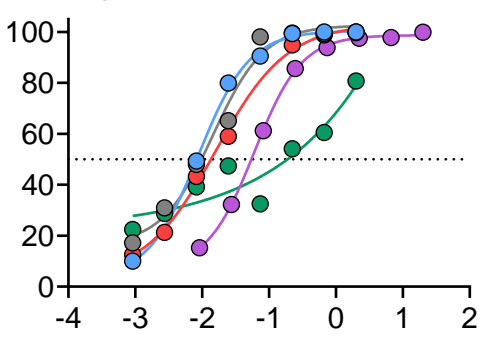

Delta

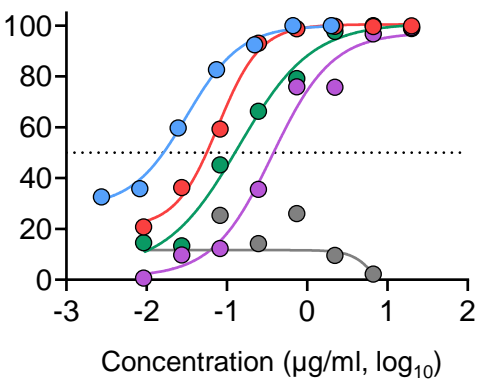

Authentic virus

Alpha

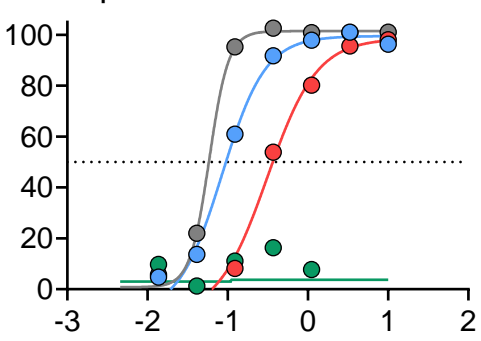

Delta

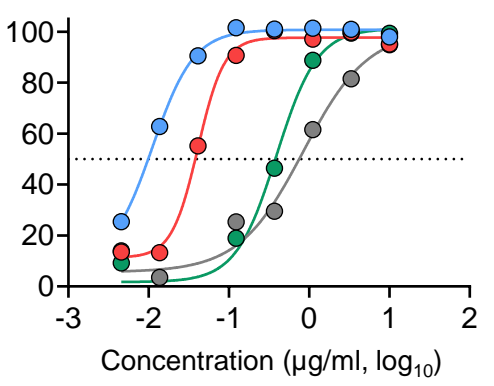

Beta

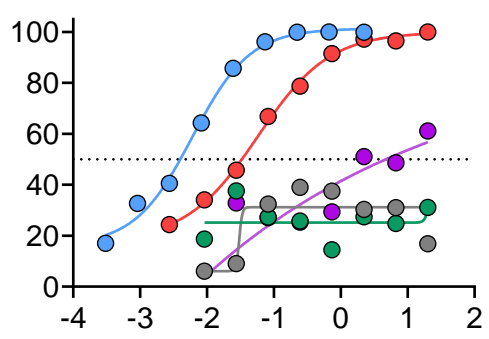

Omicron

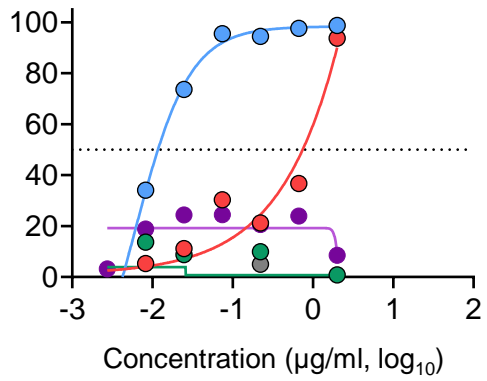

Beta

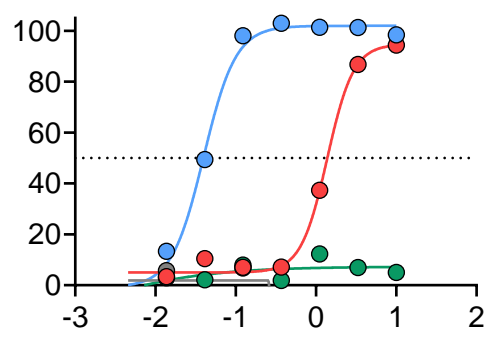

-o- Omicron

-o- Omicron $_{\text {R346K }}$

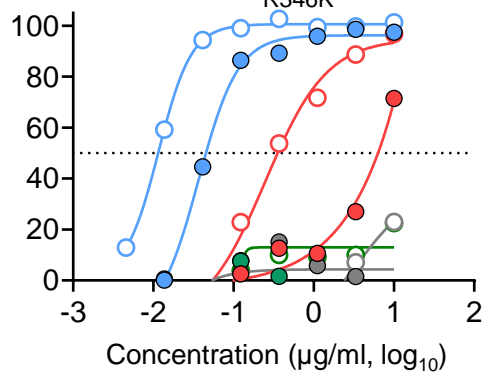




\section{Fig. 3}

a Fold-change of $I C_{50}$ relative to $D 614 G$

\begin{tabular}{|c|c|c|c|c|c|c|c|c|c|c|c|c|c|c|c|c|c|c|c|c|c|c|c|c|c|c|c|c|c|c|c|c|c|c|c|c|c|c|c|c|c|c|c|c|c|c|c|c|}
\hline \multirow{3}{*}{$\begin{array}{l}\text { Spike } \\
\text { vocs }\end{array}$} & \multirow{2}{*}{\multicolumn{20}{|c|}{ NTD }} & \multicolumn{16}{|c|}{ RBD } & & \multirow{2}{*}{\multicolumn{3}{|c|}{ SD2 }} & \multirow{2}{*}{\multicolumn{4}{|c|}{ FP }} & \multirow{2}{*}{\multicolumn{4}{|c|}{ HR1 }} \\
\hline & & & & & & & & & & & & & & & & & & & & & \multicolumn{15}{|c|}{ RBM } & & & & & & & & & & & & & \\
\hline & 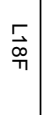 & $\begin{array}{l}-1 \\
\overrightarrow{0} \\
0 \\
\nabla\end{array}$ & $\mid \begin{array}{l}\vec{N} \\
\underline{Q} \\
\end{array}$ & 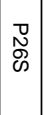 & $\stackrel{\stackrel{\leftrightarrow}{\gtrless}}{<}$ & 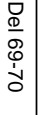 & $\begin{array}{l}\stackrel{8}{\circ} \\
\$\end{array}$ & $\begin{array}{l}\overrightarrow{0} \\
\stackrel{0}{\circ} \\
\end{array}$ & 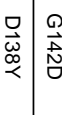 & 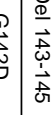 & 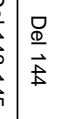 & $\begin{array}{l}\frac{m}{\vec{v}} \\
\frac{\vec{v}}{\lambda} \\
\end{array}$ & $\mid$ & 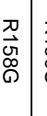 & 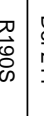 & 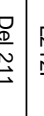 & 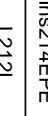 & 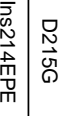 & $\begin{array}{l}0 \\
\text { N } \\
\text { N } \\
\text { N } \\
\$\end{array}$ & 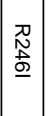 & 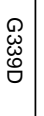 & & 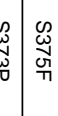 & 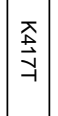 & 否 & & & $\begin{array}{l}\infty \\
\vdots \\
z \\
z\end{array}$ & $\begin{array}{l}\vec{f} \\
\text { 离 }\end{array}$ & 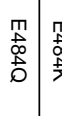 & & & 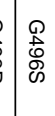 & \begin{tabular}{|l|}
0 \\
0 \\
$\mathbb{0}$ \\
$\mathbb{0}$ \\
\hdashline 0
\end{tabular} & 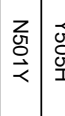 & & 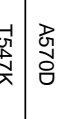 & 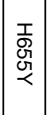 & & 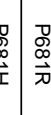 & 岁 & $\mid$\begin{tabular}{|l}
$\overrightarrow{\vec{J}}$ \\
$\underline{\partial}$
\end{tabular} & $\mid$\begin{tabular}{|}
$z$ \\
$z$ \\
多 \\
$\frac{1}{\lambda}$
\end{tabular} & 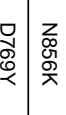 & $\mid \begin{array}{l}\vec{T} \\
0 \\
\underline{Z} \\
\underline{z}\end{array}$ & & & 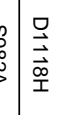 \\
\hline Alpha & & & & & & & & & & & & & & & & & & & & & & & & & & & & & & & & & & & & & & & & & & & & & & & & \\
\hline Beta & & & & & & & & & & & & & & & & & & & & & & & & & & & & & & & & & & & & & & & & & & & & & & & & \\
\hline Gamma & & & & & & & & & & & & & & & & & & & & & & & & & & & & & & & & & & & & & & & & & & & & & & & & \\
\hline Delta & & & & & & & & & & & & & & & & & & & & & & & & & & & & & & & & & & & & & & & & & & & & & & & & \\
\hline Omicron & & & & & & & & & & & & & & & & & & & & & & & & & & & & & & & & & & & & & & & & & & & & & & & & \\
\hline & & & & & & & & & & & & & & & & & & & & & & & & & & & & & & & & & & & & & & & & & & & & & & & & \\
\hline ZCB3 & 8 & 3 & 2 & 3 & -2 & 2 & 7 & 2 & \begin{tabular}{l|l}
2 & 12
\end{tabular} & \begin{tabular}{l|l|l}
2 & -2
\end{tabular} & $\begin{array}{ll}2 & 1\end{array}$ & 2 & 2 & 2 & 3 & -1 & 1 & \begin{tabular}{l|l}
4 & -1
\end{tabular} & 1.4 & \begin{tabular}{|l|}
1.4 \\
\end{tabular} & -1 & \begin{tabular}{l|l}
1 & 5
\end{tabular} & \begin{tabular}{l|l}
.4 & 1.1
\end{tabular} & 12 & \begin{tabular}{|l|l|}
2.6 & 1
\end{tabular} & \begin{tabular}{l|l}
1.4 & 1
\end{tabular} & $\begin{array}{lll}1.2 & 2 .\end{array}$ & $\begin{array}{l}7 \\
\end{array}$ & -1 & \begin{tabular}{|l|l|l} 
& 2
\end{tabular} & $\begin{array}{l}2.3 \\
-\end{array}$ & \begin{tabular}{|l|l|l}
-1 & -2
\end{tabular} & -2 & 1.1 & \begin{tabular}{|l|l|}
-1.6 & -2
\end{tabular} & \begin{tabular}{l|l|l}
-2 & 1.6
\end{tabular} & \begin{tabular}{l|l|l}
.6 & 3.5
\end{tabular} & $2.5 \mid$ & \begin{tabular}{l|l}
-2 & 3
\end{tabular} & .92 .6 & 4.8 & \begin{tabular}{|l|}
1.4 \\
\end{tabular} & \begin{tabular}{|l|l|}
-1 & -2
\end{tabular} & \begin{tabular}{l|l}
-2 & 2.6
\end{tabular} & $\begin{array}{l}5 \\
\end{array}$ & \begin{tabular}{|l|l|}
-2 & 1 \\
\end{tabular} & & \begin{tabular}{l|l}
.3 & 2.3 \\
\end{tabular} \\
\hline ZCB11 & -2 & -1 & 2 & -1 & -4 & -3 & -1 & 3 & \begin{tabular}{l|l}
1 & 1
\end{tabular} & -4 & $\begin{array}{l}4 \\
4\end{array}$ & 1 & 1 & 1 & -2 & -2 & -3 . & \begin{tabular}{l|l}
-4 & 4.7
\end{tabular} & -2 & 1.2 & -3 & \begin{tabular}{ll|l}
-11 & 1
\end{tabular} & \begin{tabular}{l|l}
3 & -2
\end{tabular} & $|-1|$ & $-3 \mid$ & \begin{tabular}{l|l}
-2 & -
\end{tabular} & \begin{tabular}{l|l}
-2 & 1.
\end{tabular} & \begin{tabular}{c|c|}
4 & -5 \\
\end{tabular} & -4 & -2 & -2 & -4 & -3 & -3 & \begin{tabular}{|l|l|}
-1.2 & -7
\end{tabular} & 7 & \begin{tabular}{l|l}
6 & -2
\end{tabular} & $3.7 \mid$ & \begin{tabular}{l|l}
-5 & -
\end{tabular} & \begin{tabular}{l|l}
2 & 1.4
\end{tabular} & & \begin{tabular}{|l|}
2.4 \\
\end{tabular} & \begin{tabular}{|l|l|} 
& -5 \\
\end{tabular} & \begin{tabular}{l|l}
-2 & 1.1
\end{tabular} & 1.2 & -9 & \begin{tabular}{l|l}
-1 & 2.
\end{tabular} & \begin{tabular}{l|l}
9 & 1
\end{tabular} \\
\hline ZB8 & 1 & 3 & 4 & 2 & -3 & -2 & 3 & 1 & \begin{tabular}{l|l}
5 & 1
\end{tabular} & -3 & \begin{tabular}{l|l}
3 & -2
\end{tabular} & 2 & 1 & 4 & 2 & -3 & -4 & \begin{tabular}{l|l}
-2 & -3
\end{tabular} & -2 & \begin{tabular}{|l|l|} 
\\
\end{tabular} & -2 & \begin{tabular}{|l|l|l}
-4 &
\end{tabular} & \begin{tabular}{l|l}
2 & -1
\end{tabular} & 1.4 & $-3 \mid$ & \begin{tabular}{l|l}
-2 & -
\end{tabular} & \begin{tabular}{l|l}
-4 & -5
\end{tabular} & \begin{tabular}{l|l|}
4 & -3
\end{tabular} & $\mid-2$ & & & -1 & -3 & -2 & \begin{tabular}{|l|l|l|} 
& -1.7
\end{tabular} & \begin{tabular}{l|l}
-8 & -1
\end{tabular} & \begin{tabular}{l|l}
1 & 1.8
\end{tabular} & 1.7 & \begin{tabular}{l|l}
-3 & 1
\end{tabular} & \begin{tabular}{l|l|l|}
3 & 1.4
\end{tabular} & -2 & 4.5 & \begin{tabular}{|l|l|}
-4 & -2
\end{tabular} & \begin{tabular}{l|l}
-2 & -1
\end{tabular} & 1.7 & -6 & -2 & \begin{tabular}{l|l}
3 & 1.3
\end{tabular} \\
\hline
\end{tabular}

b

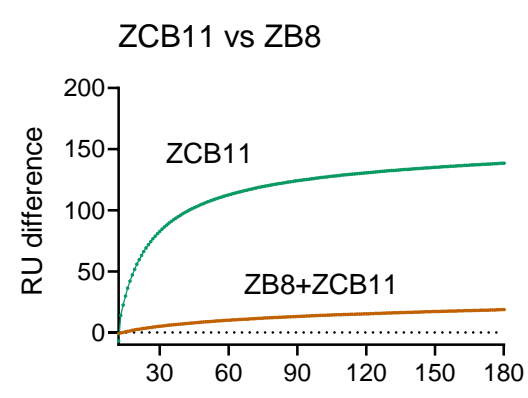

ZCB11 vs ZB3

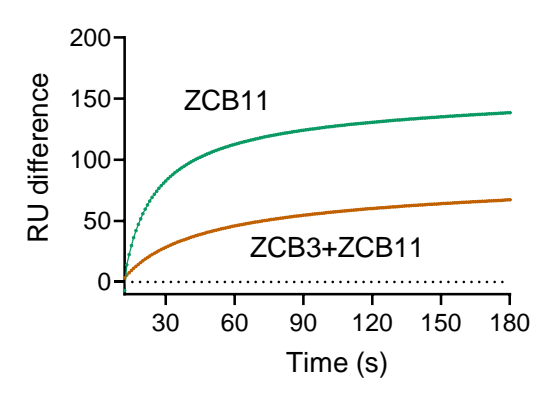

C
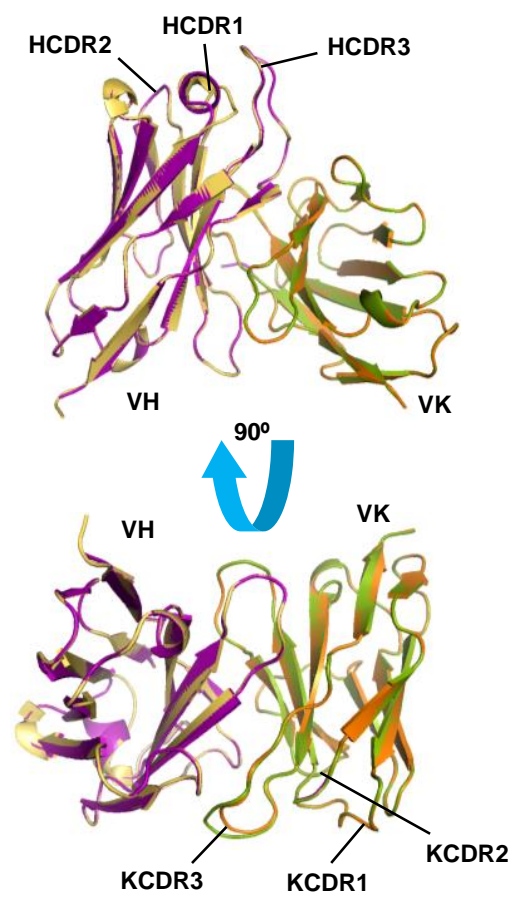

d

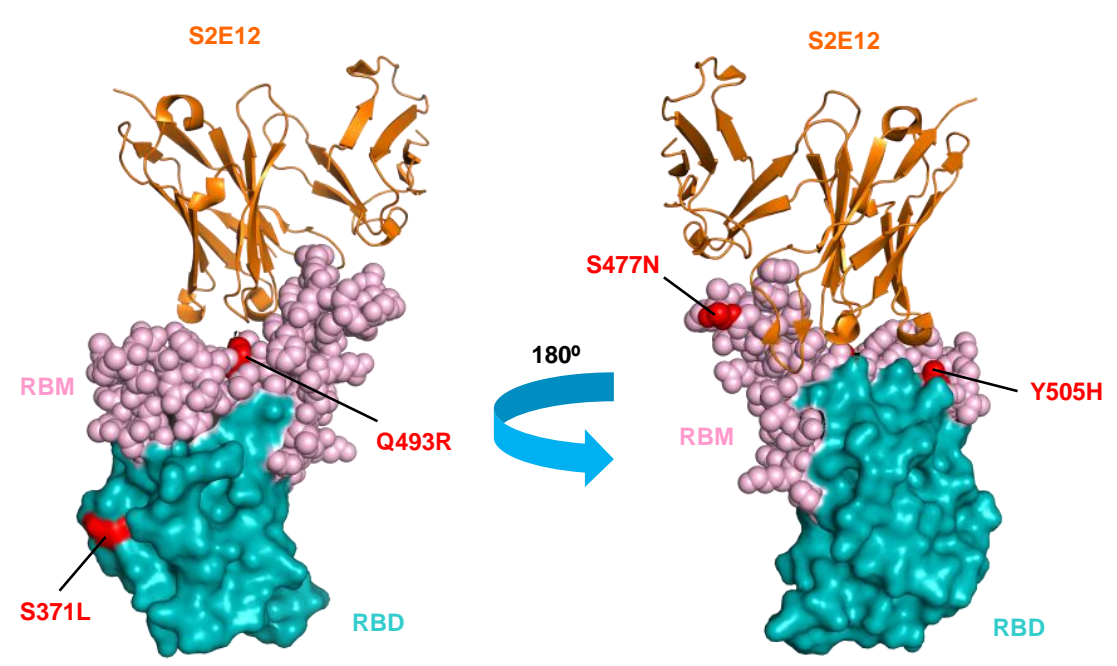


a

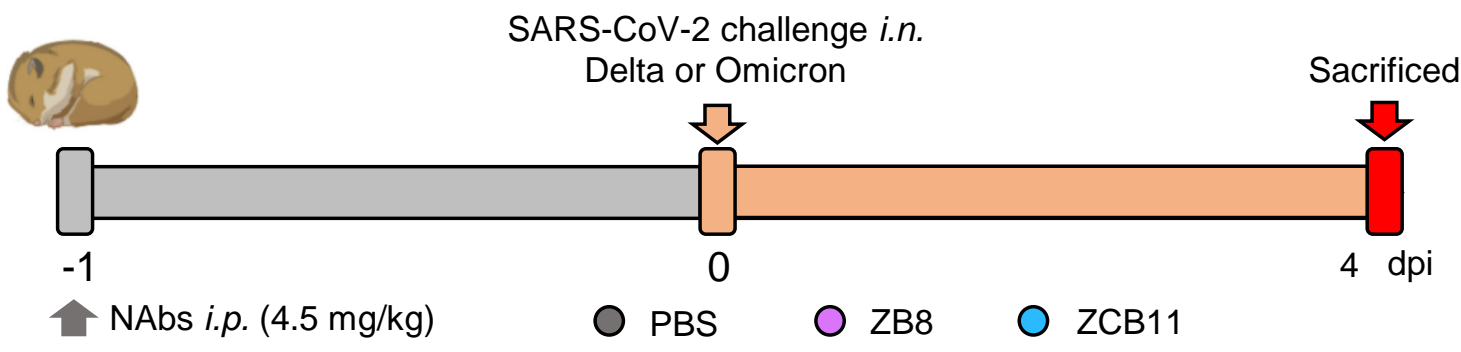

C

Delta b

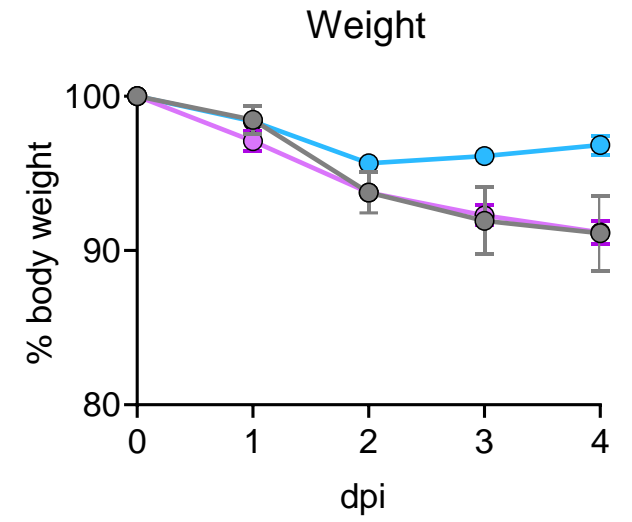

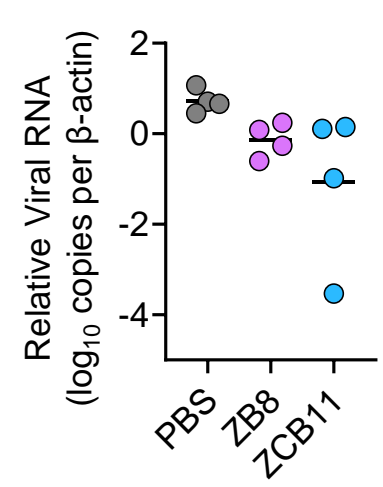

d

Infectious virus

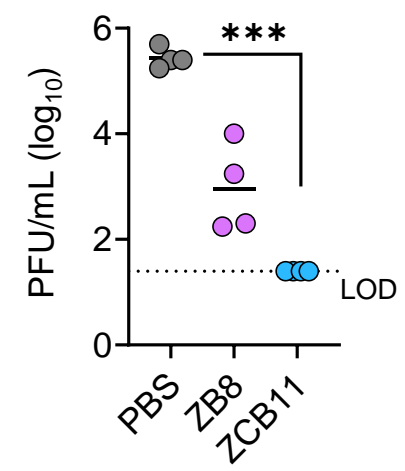

Omicron

e

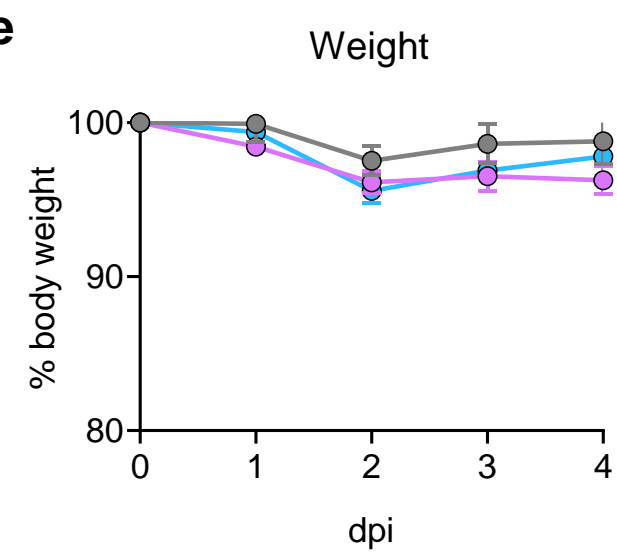

f

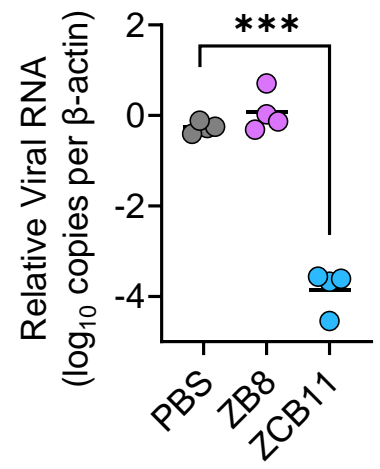

g Infectious virus

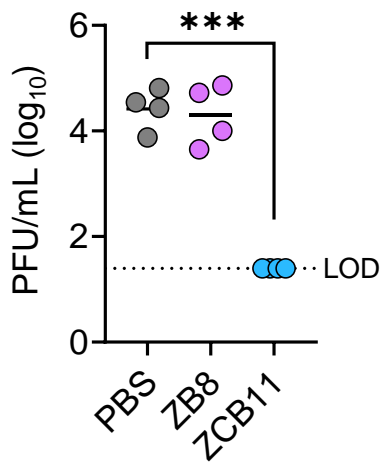

